\title{
Landsat 9 Thermal Infrared Sensor 2 Pre-Launch Characterization: Initial Imaging and Spectral Performance Results
}

Aaron Pearlman ${ }^{1}$, Joel McCorkel ${ }^{2}$, Matthew Montanaro ${ }^{3}$, Boryana Efremova $^{1}$, Brian Wenny ${ }^{4}$, Allen Lunsford ${ }^{5}$, Amy Simon ${ }^{2}$, Jason Hair $^{2}$, and Dennis Reuter ${ }^{2}$

${ }^{1}$ GeoThinkTank LLC, ${ }^{2}$ NASA Goddard Space Flight Center, ${ }^{3}$ Rochester Institute of Technology, ${ }^{4}$ Science Systems and Applications, ${ }^{5}$ Catholic University of America

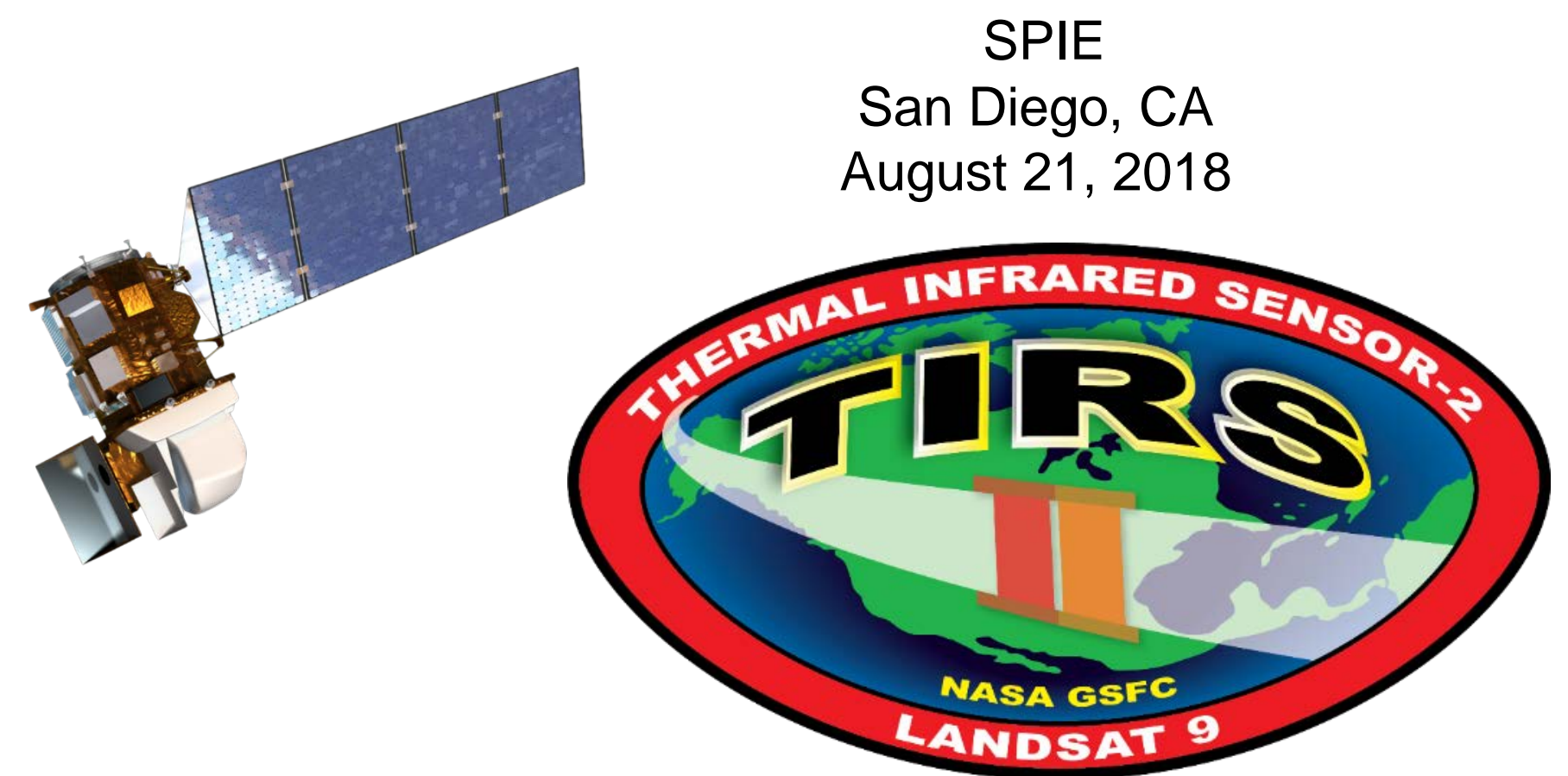




\section{TIRS-2 Project Overview}

- TIRS-2 will fly on the LandSat 9

- 16 day re-visit cycle

- 2 bands: $10.8 \mu \mathrm{m} \& 12 \mu \mathrm{m}$

- Like TIRS on Landsat 8, TIRS-2 will produce radiometrically calibrated, geo-located thermal image data

- Risk Class C for Landsat 8 to Class B for Landsat 9

- Increased redundancy to satisfy Class B reliability standards

- Improved stray light performance through improved telescope baffling

- Improved position encoder for scene select mirror to address problematic encoder on Landsat 8 TIRS

- USGS will be responsible for operations

- TIRS-2 development:

- NASA GSFC TIRS-2 team formed in 2015

- TIRS-2 completed Critical Design Review in Feb. 2017

- Instrument in fabrication at NASA GSFC

- Initial pre-launch imaging and spectral characterization Nov. 2017 - March 2018

- On target for August-2019 delivery to spacecraft
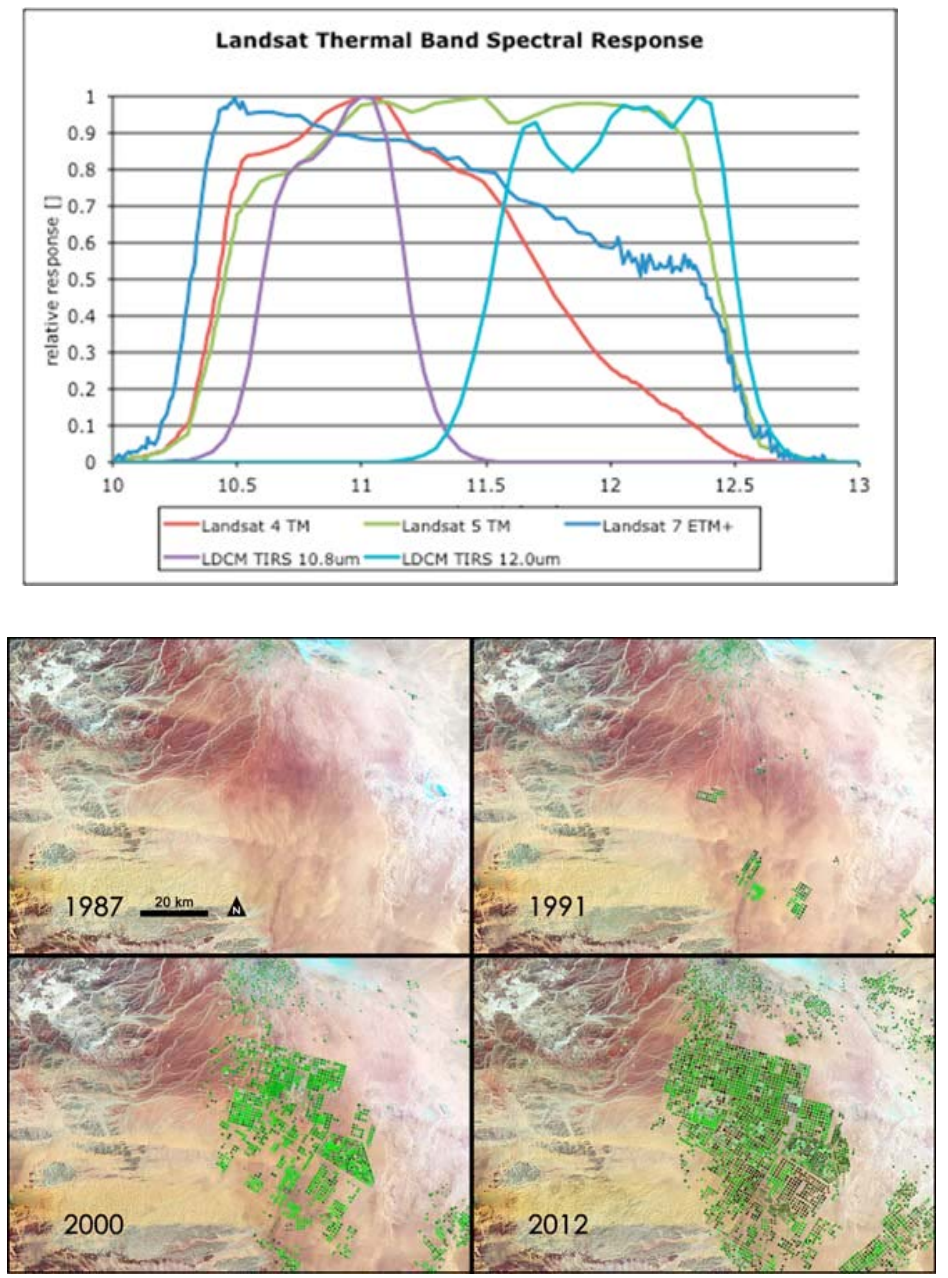

-Increase in pivot irrigation in Saudi Arabia from 1987 to 2012 as recorded by Landsat. The increase in irrigated land correlates with declining groundwater levels measured from GRACE (courtesy M. Rodell, GSFC) 


\section{Stray Light Issue from TIRS}

- Non-uniform banding and absolute calibration error found in TIRS imagery post-launch - suspected stray light

- Characterized on-orbit using a rasterscan of the moon around the out-offield-view

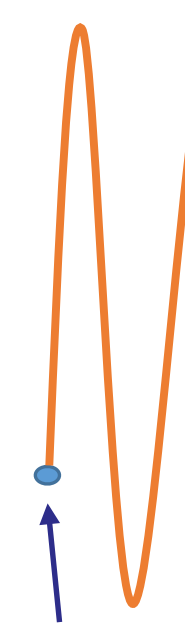

Moon is

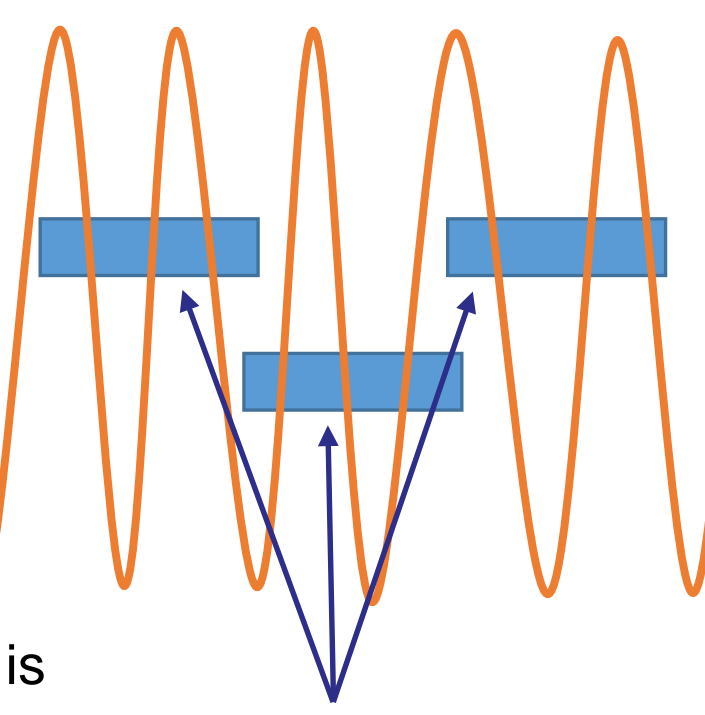

$\sim 0.5$ deg. wide

TIRS detector arrays

M. Montanaro. et al. Remote Sensing, (2014).
Stray light source roughly $13^{\circ}$ from optical axis
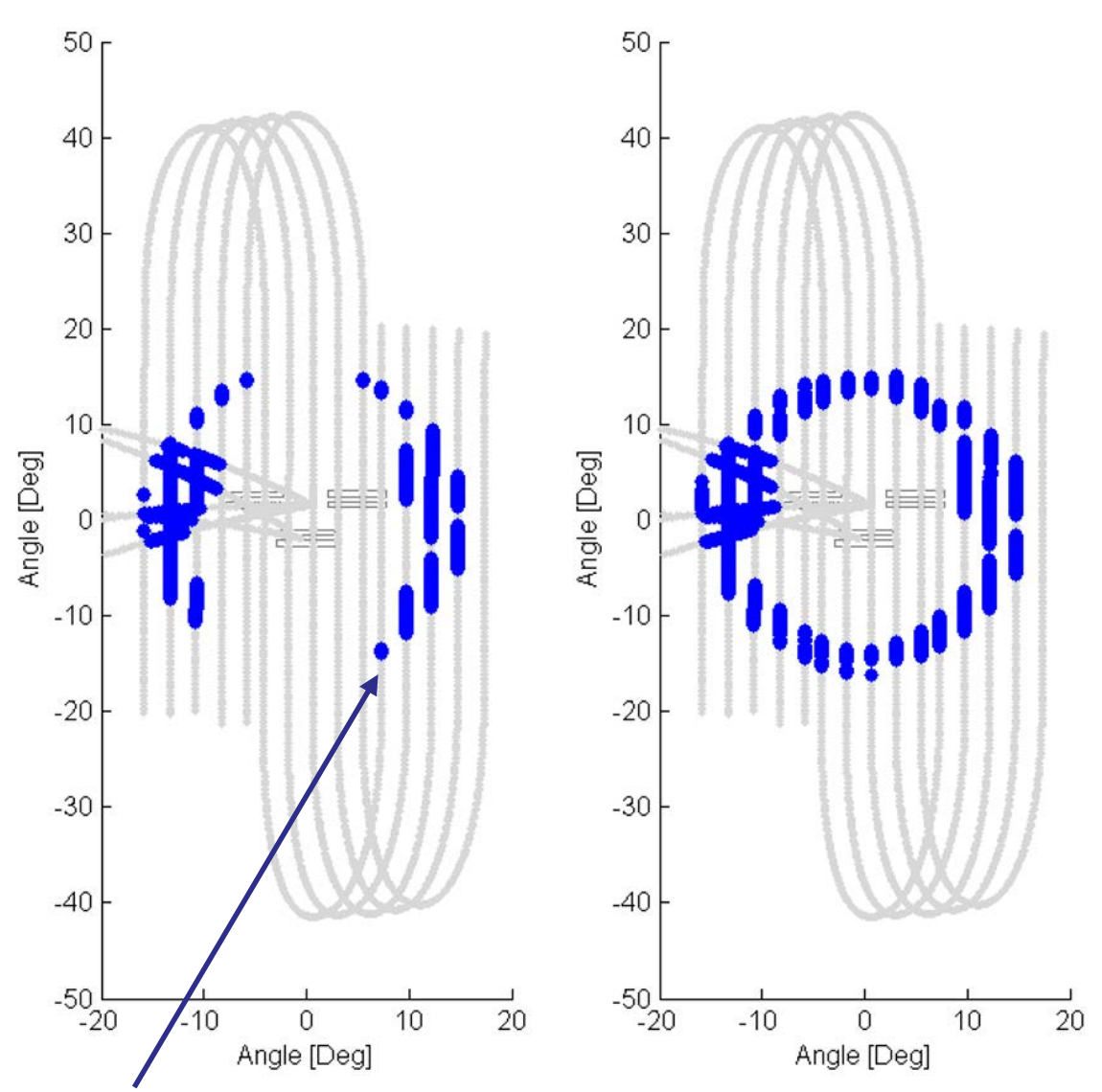

Lunar locations where a stray light signal appeared anywhere on the detectors 


\section{TIRS-2 Architecture}

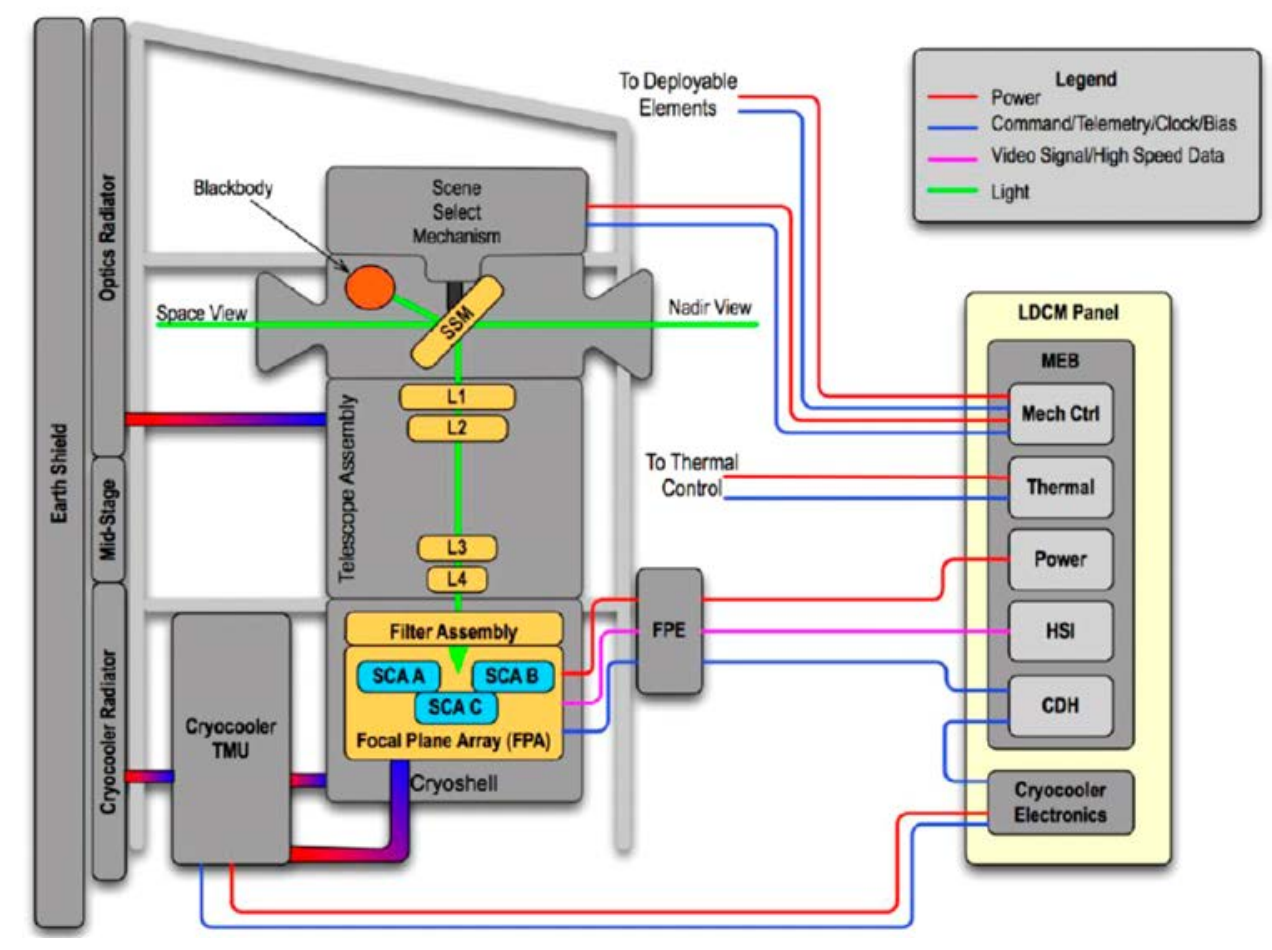




\section{TIRS-2 Architecture}

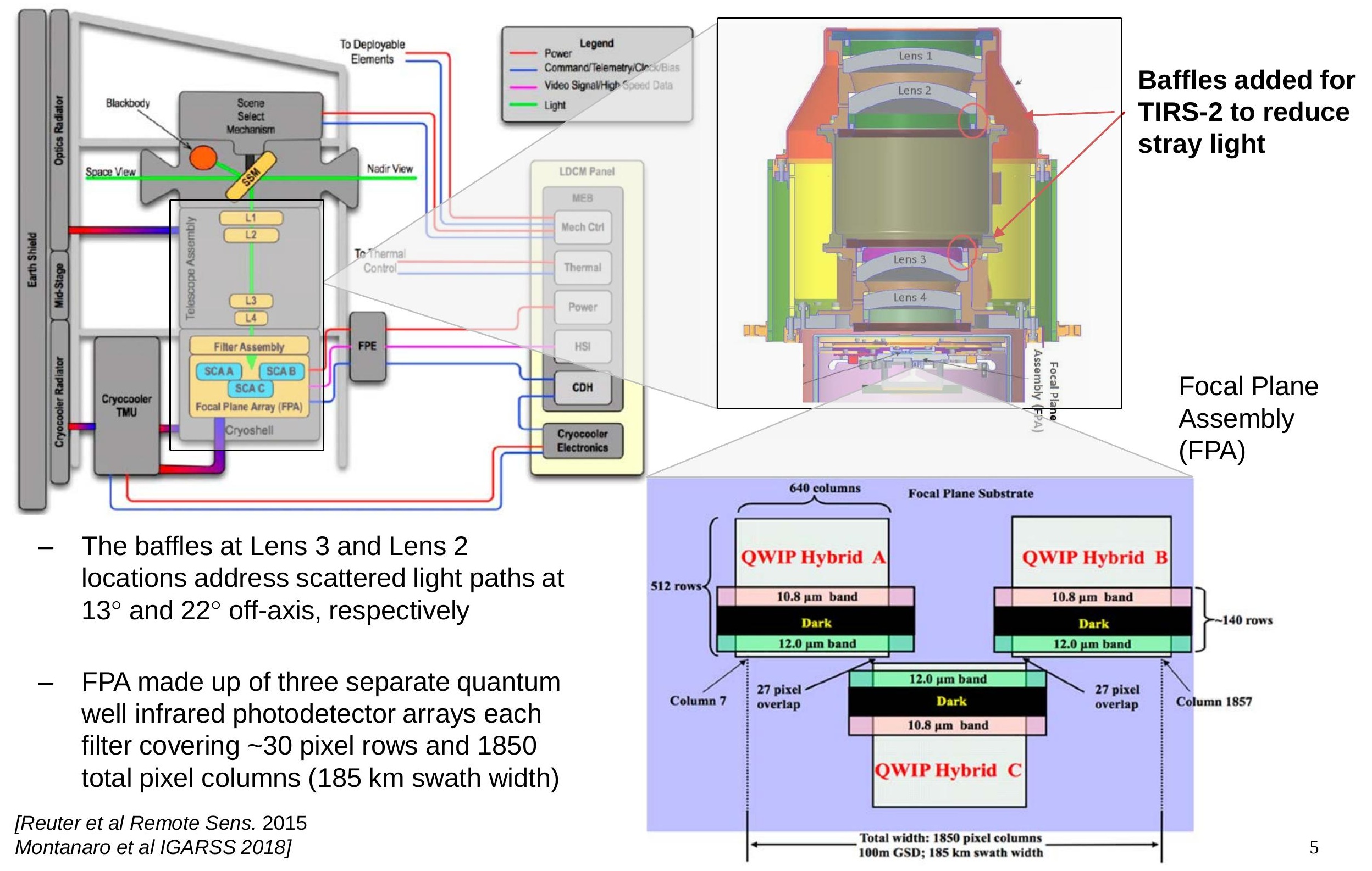


Initial Pre-Launch Imaging and Spectral Characterization: aka TIRS-2 Imaging Performance \& Cryoshell Evaluation (TIPCE)

Initial performance tests at "almost" instrument-level (Telescope/focal plane arrays/focal plane electronics, no scene select mirror)

- Focus test

- Determine focus position of FPA/telescope, determine proper shims, \& verify

- Spatial response test - Initial characterization

- Scatter survey test

- Only opportunity to measure far-field scattering (due to config of test article and CGSE in the chamber)

- Spectral response test - Initial characterization

- Characterize cryoshell performance 


\section{TIPCE Configuration Focus, Scatter, \& Spatial}

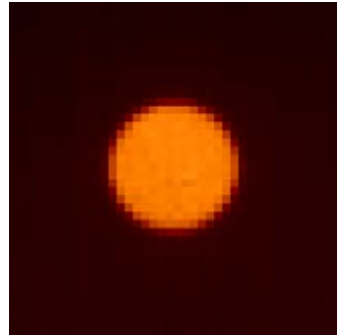

16-pixel circular target

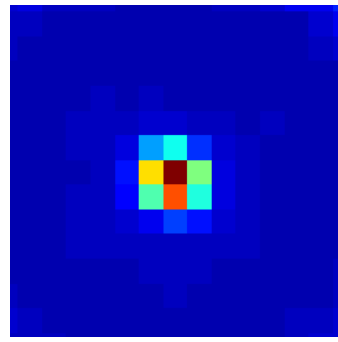

1- and 2-pixel circular targets

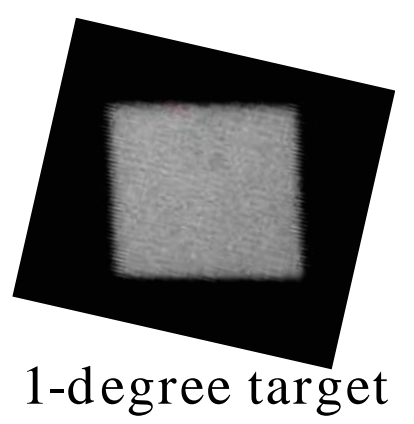

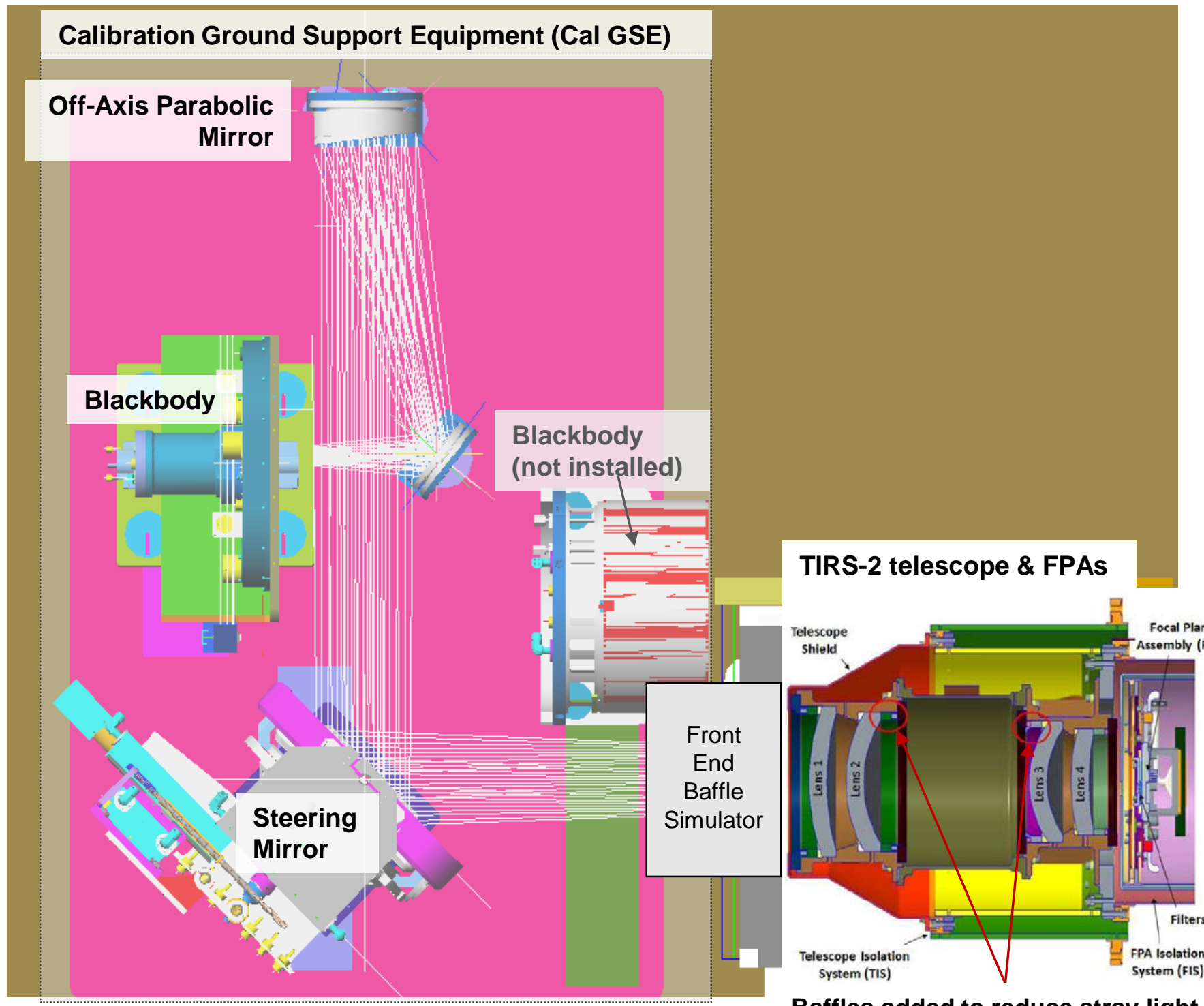

Baffles added to reduce stray light 


\section{Focus Test Methodology}

- The Focus Test is used to determine the optimal focus position of the TIRS-2 focal plane assembly (FPA) relative to the optical telescope.

- Optimal focus is determined by minimizing the full-width, half-maximum (FWHM) of a Gaussian-based model fit to the image created by an input twopixel source.

- This focus map is then reported to the instrument team so that proper shims can be fabricated and installed.

- These measurements are first performed at the telescope-FPA assembly (TIPCE level) to find best focus, then repeated at the full instrument level to validate consistency and characterize focus as function of telescope temperature.
Two-Pixel Source
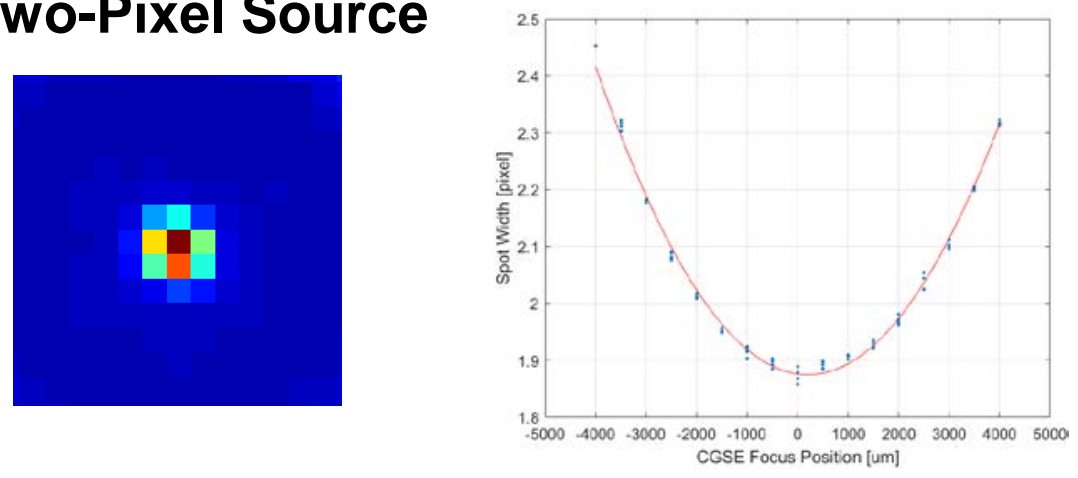

Focus Test Methodology

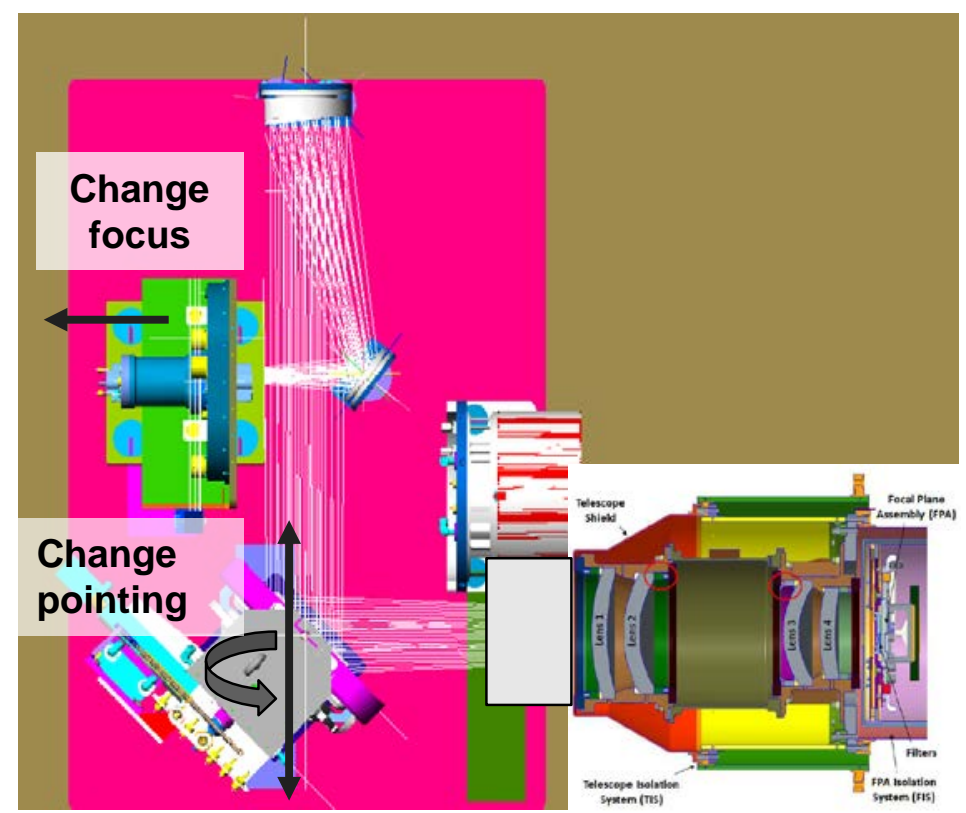




\section{Focus Test Results}

- Full focus survey collected during TIPCE with telescope at nominal temperature

- Shims calculated, manufactured, and installed

- Full focus survey for verification collected during another phase of TIPCE at nominal telescope temperature and at nominal $+5 \mathrm{~K}$.

- Found average piston defocus of +90 microns of CGSE z-axis

- shim deltas to be only: $+0.0003 ",+0.0002 ",-0.0002 "$

- Decided on NO shim adjustment

- Decided on NO telescope temperature adjustment
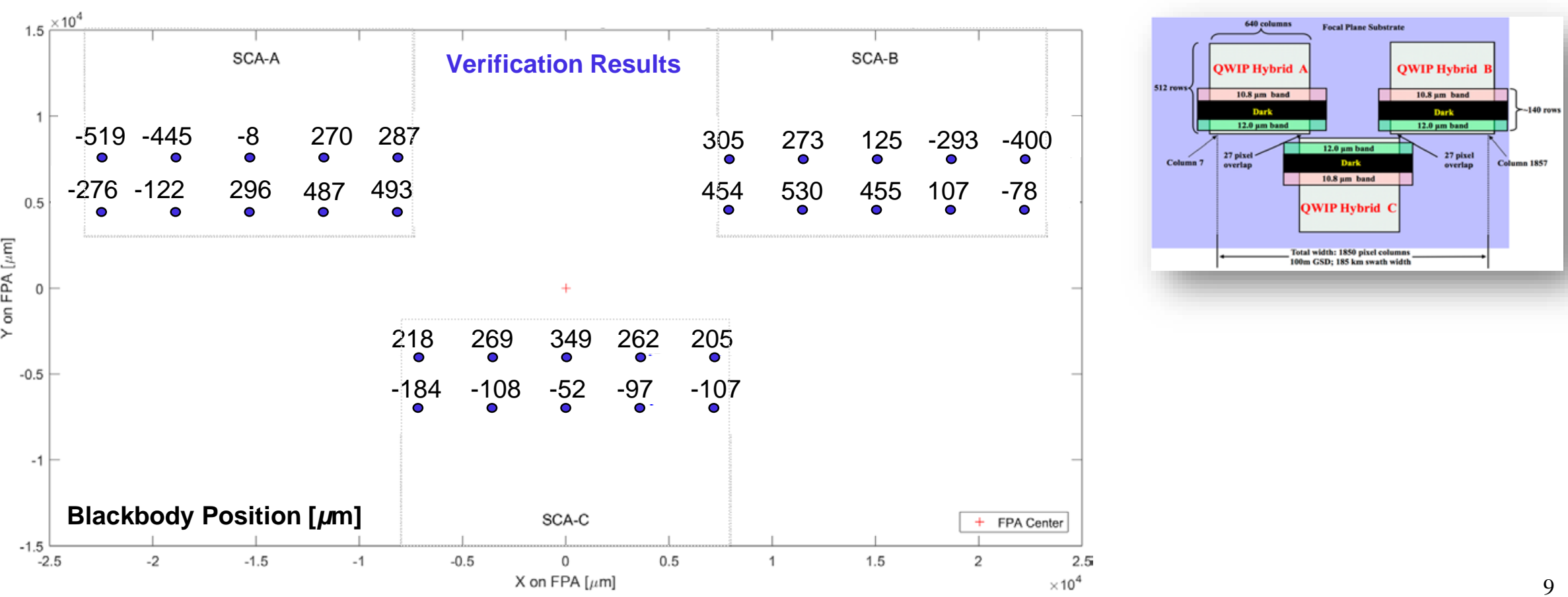

$$
\begin{array}{ccccc}
218 & 269 & 349 & 262 & 205 \\
0 & 0 & 0 & 0 & 0 \\
-184 & -108 & -52 & -97 & -107 \\
0 & 0 & 0 & 0 & 0
\end{array}
$$




\section{Spatial Response Test Methodology}

[Wenny et al. Remote Sens. (2015)]

\section{- Processing follows the same methodology as used for TIRS1}

- Using 'hockey puck' target collect frames as target is moved in incremental sub-pixel (1/5) steps across-track and along-track over 3 pixels in each direction.

- 16 pixel diameter circle target ("Hockey Puck")

- Large square for flat field

- Blank for background correction

- Repeat at different locations on FPA

Raw image of 'hockey puck'

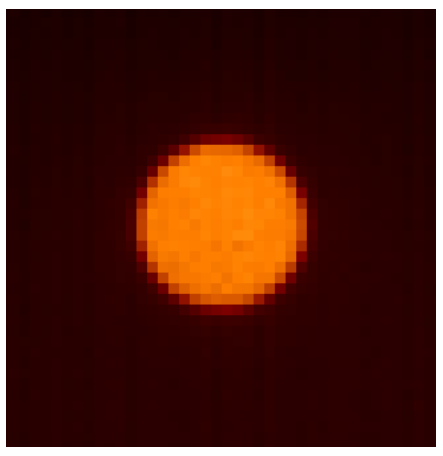

Locations of collections

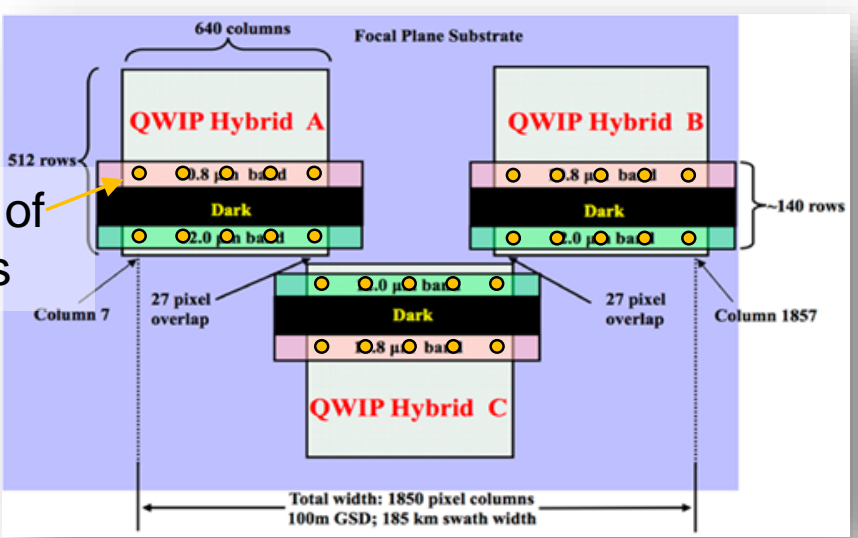

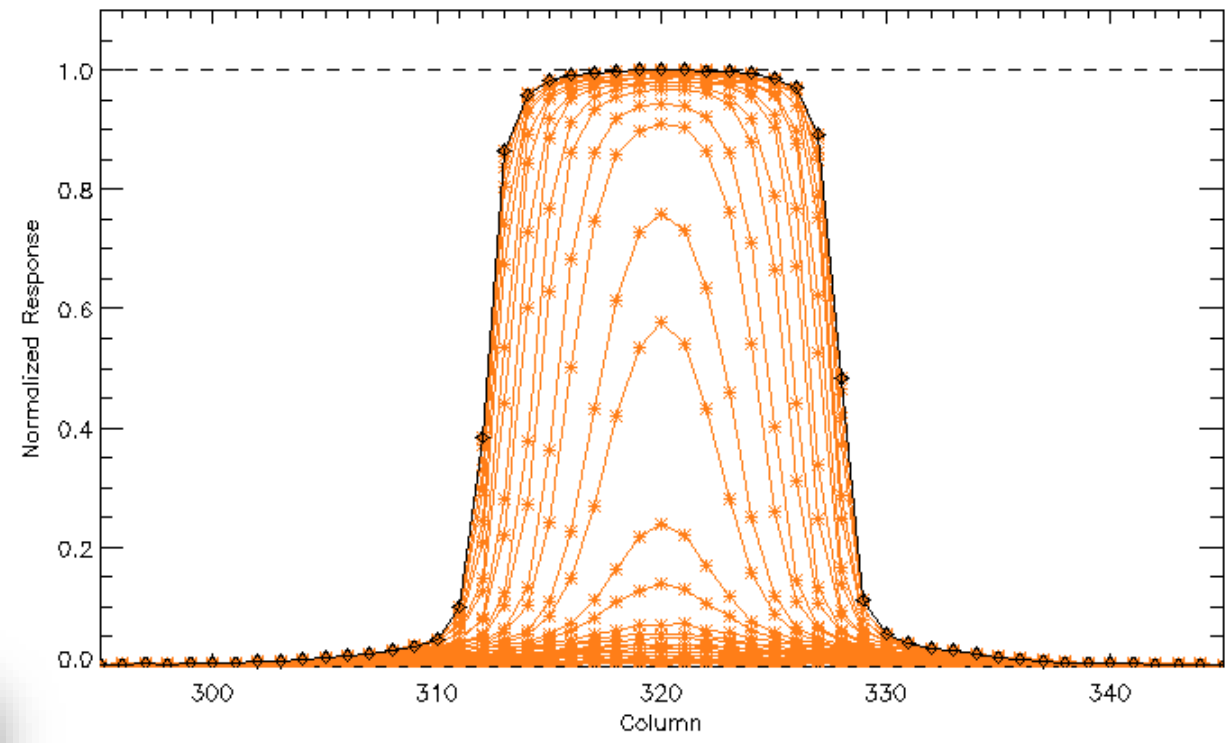

Each circular image frame has a background-correction and flat field applied at pixel level

$$
\operatorname{dn}(\mathrm{i}, \mathrm{j})=\left(D N_{P}(\mathrm{i}, \mathrm{j})-\mathrm{DN}_{\mathrm{BKG}}(\mathrm{i}, \mathrm{j})\right) /\left(\mathrm{DN}_{\mathrm{FF}}(\mathrm{i}, \mathrm{j})-\mathrm{DN}_{\mathrm{BKG}}(\mathrm{i}, \mathrm{j})\right)
$$

Horizontal cross section through center of puck normalized to maximum value 


\section{Spatial Response Test Methodology}

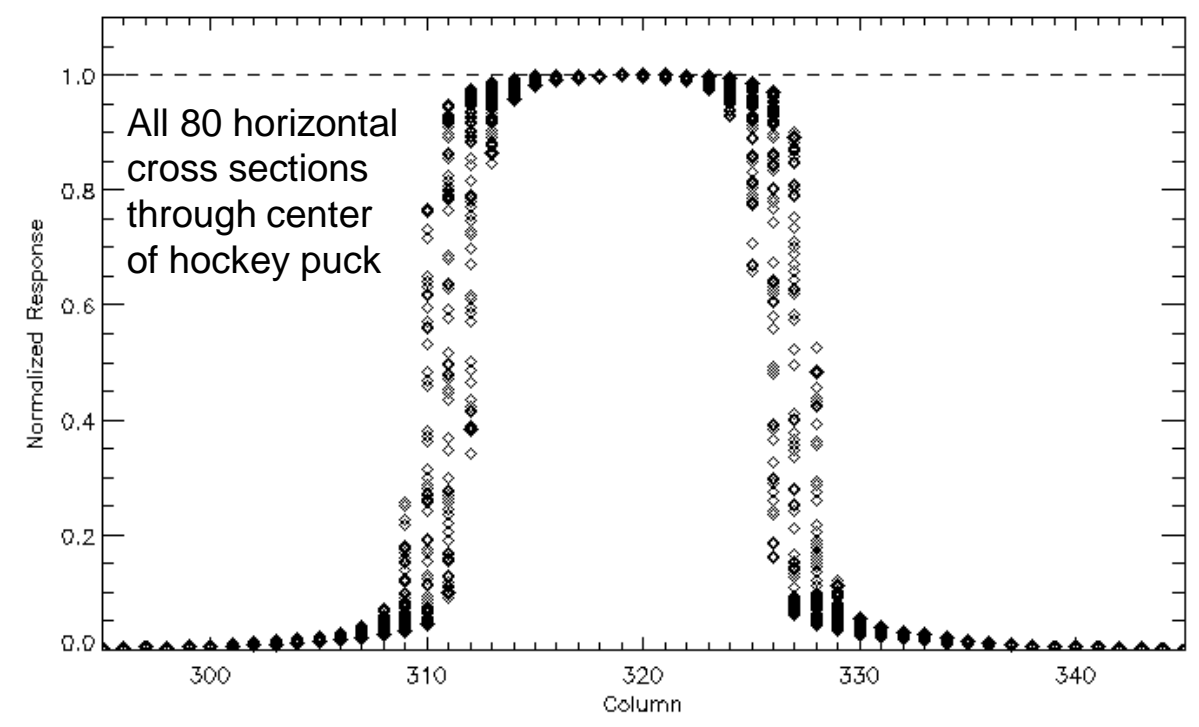

All 80 cross sections shifted to common reference point

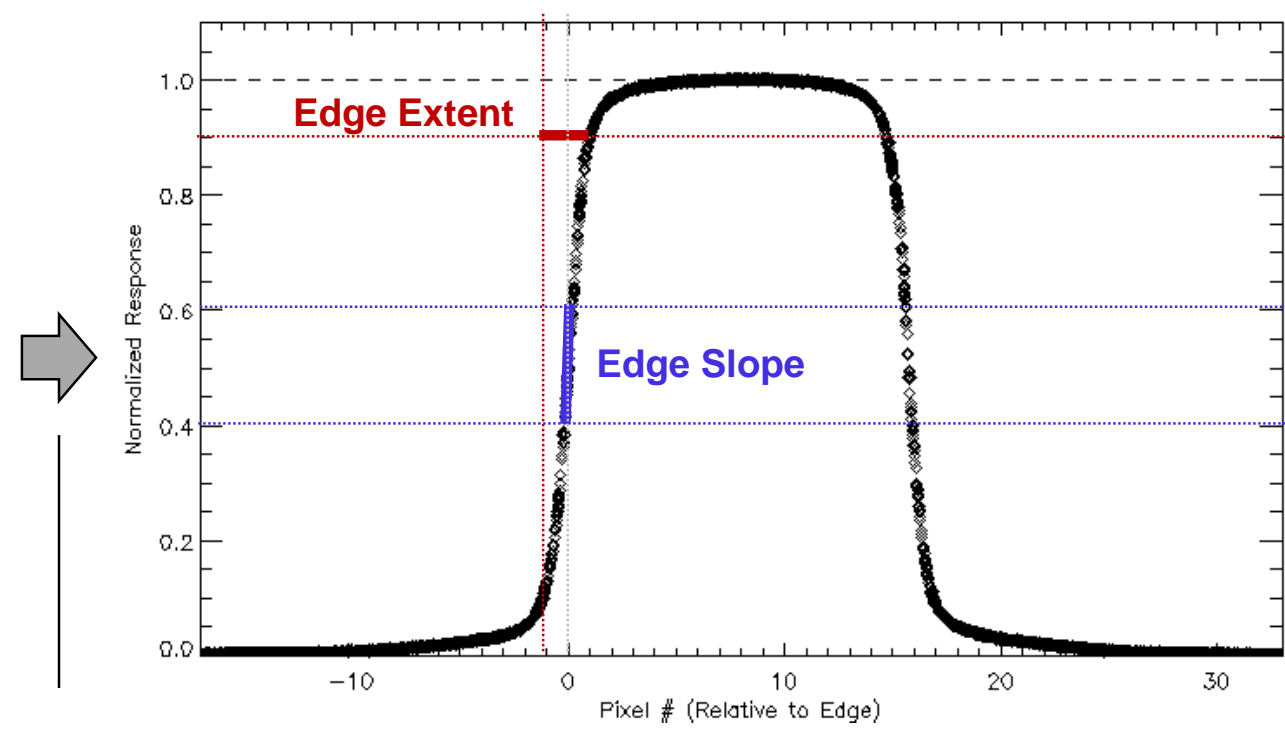
Each frame fit with Fermi function to derive edge midpoint:

$$
f(x)=\frac{a}{\left(e^{(x-b) / c}\right)+1}+d
$$
Each cross section shifted to match up mid- points resulting in a well populated edge

Metrics for evaluating spatial performance -- edge slope, edge extent -derived from each edge response plot. 


\section{Scatter Results: Target @ -28 deg}

Blackbody square target is here

Frame of signal corresponding to the grid location
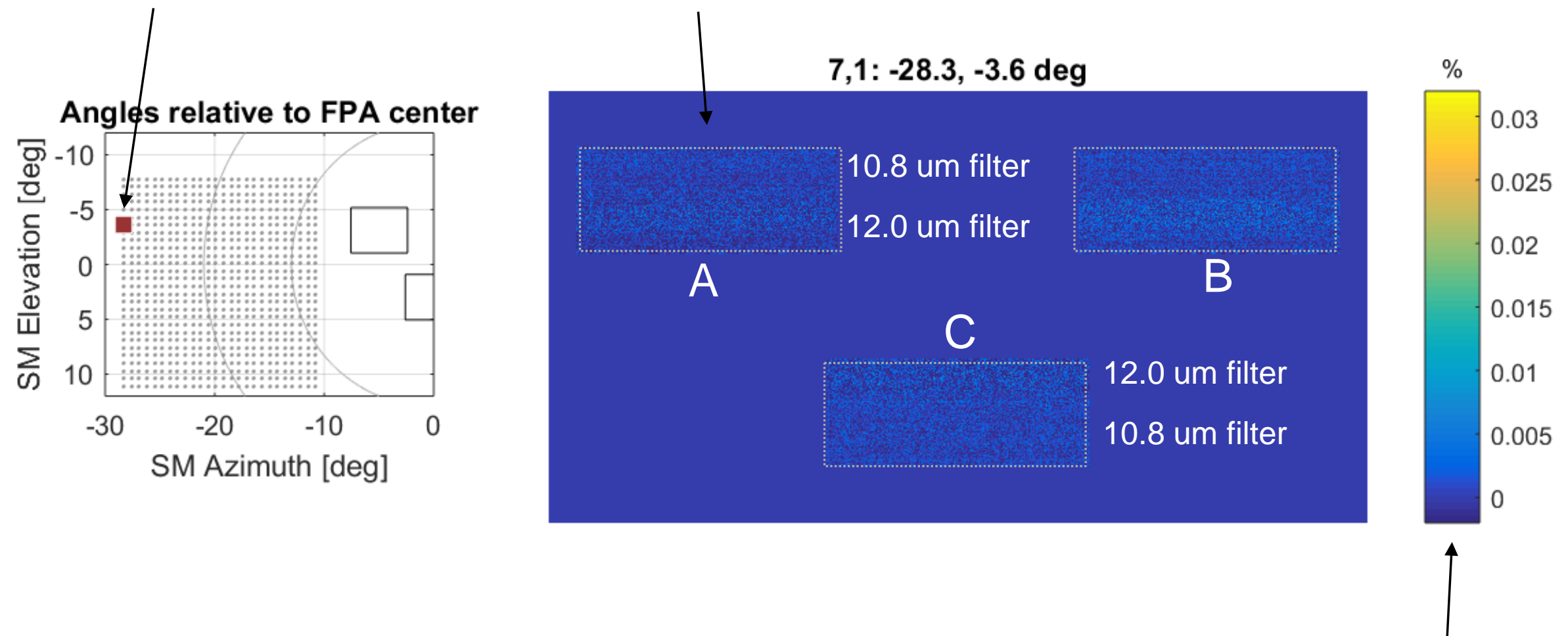

Units are percent of the signal when the target is directly illuminated on the detectors 


\section{Scatter Results: \\ Target @ -22 deg and @-13 deg}

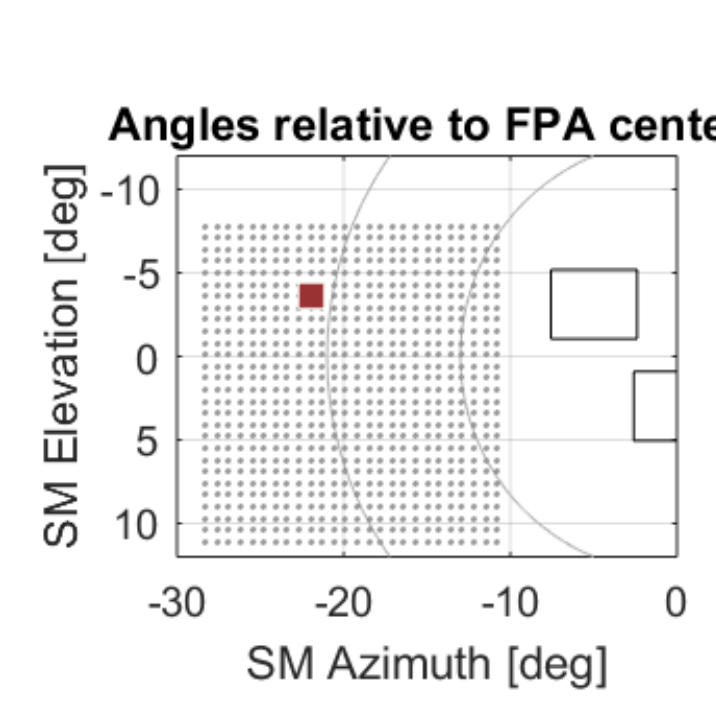

7,10: -22.0, -3.6 deg
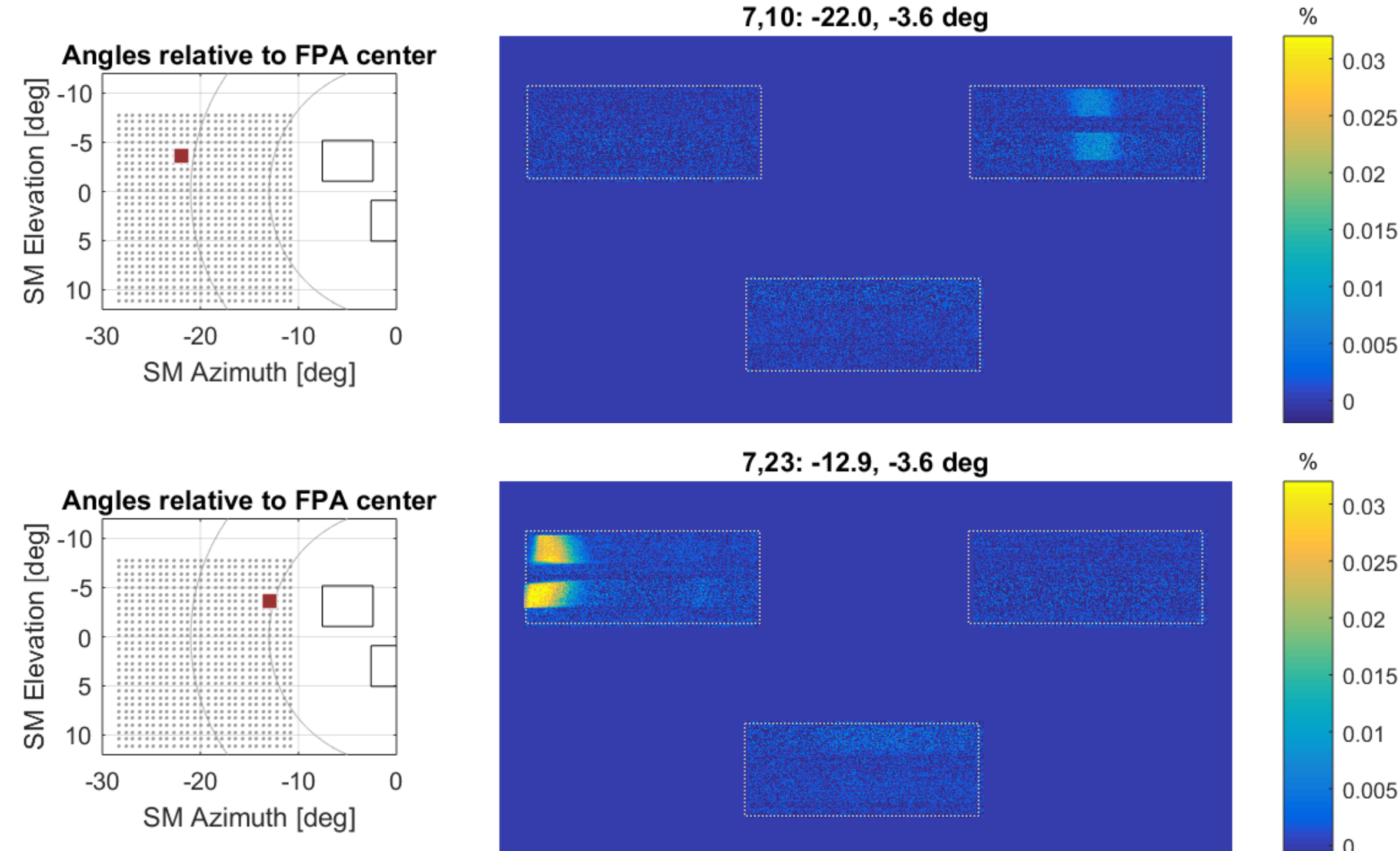

7,23: -12.9, -3.6 deg

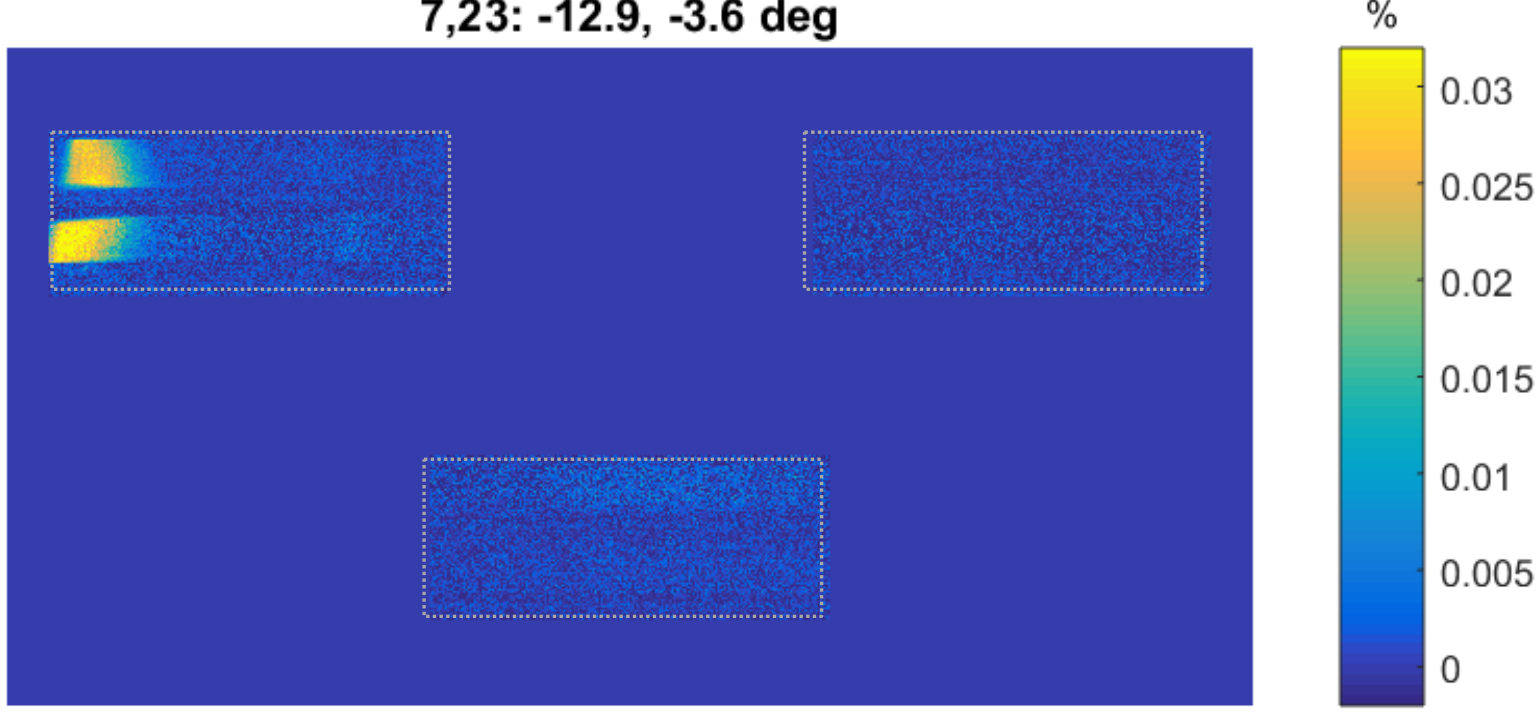




\section{Scatter Results: Total Scattering}

- Combine scattering data from TIPCE2 and TIPCE3. Red boxes where source was when signal observed on any detector.

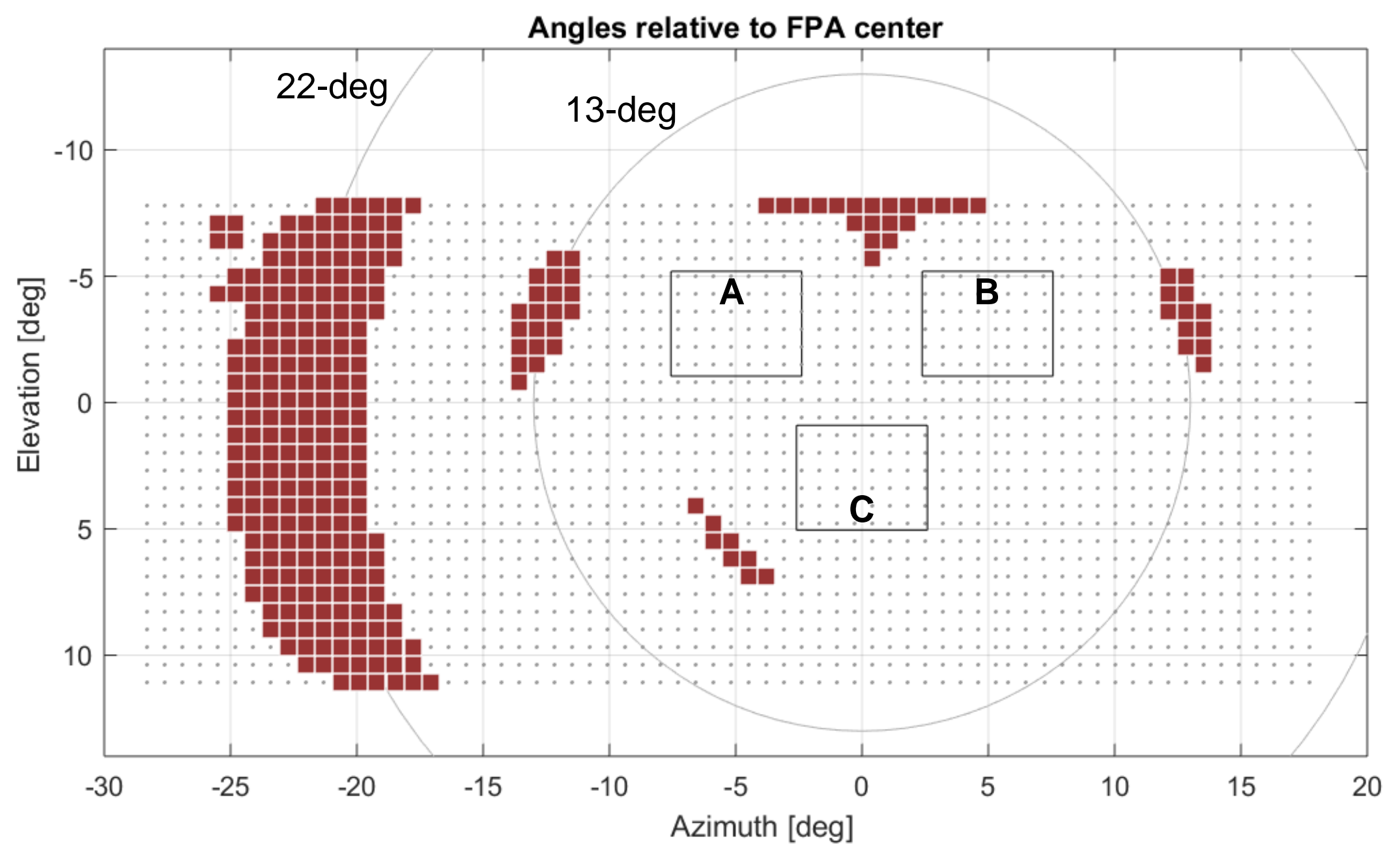




\section{Scatter Results: TIPCE Scattering Sum}

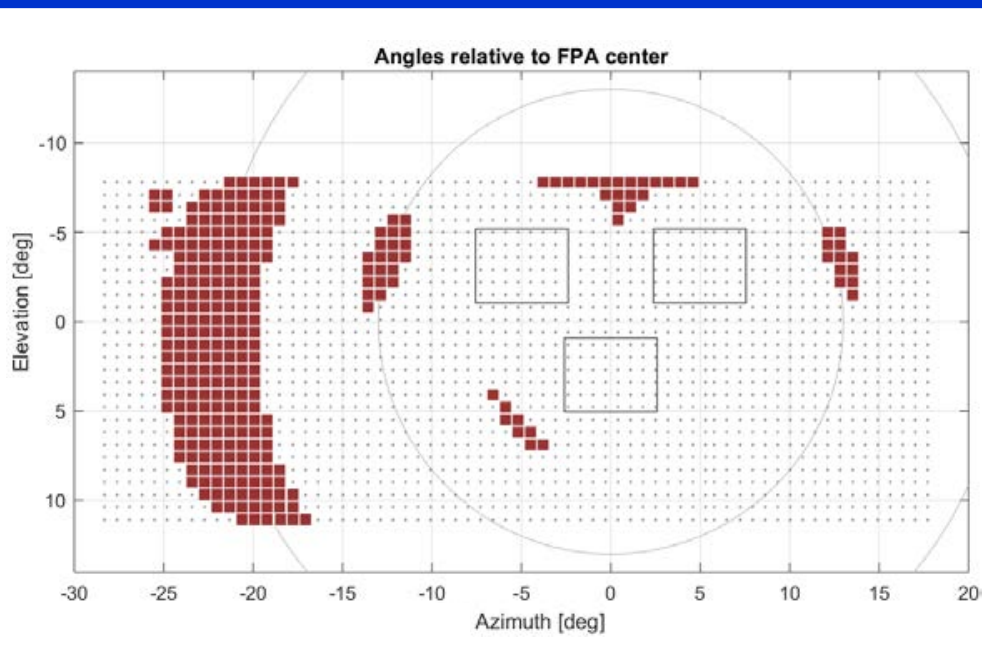

Percent signal
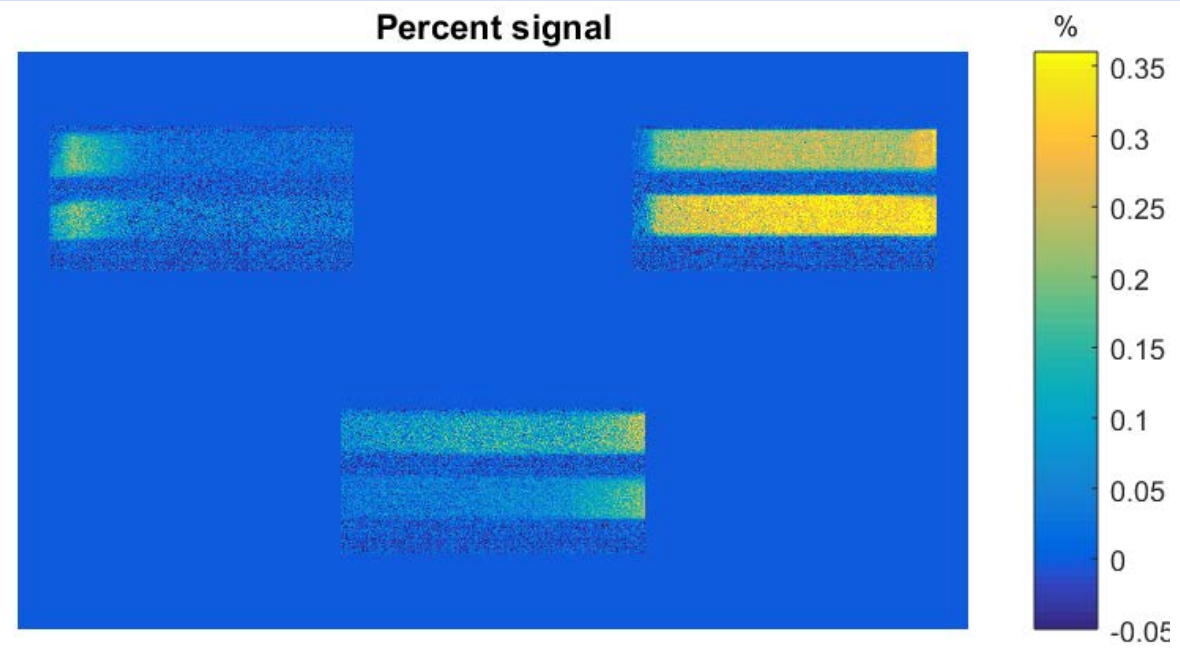

Signal profile through each filter

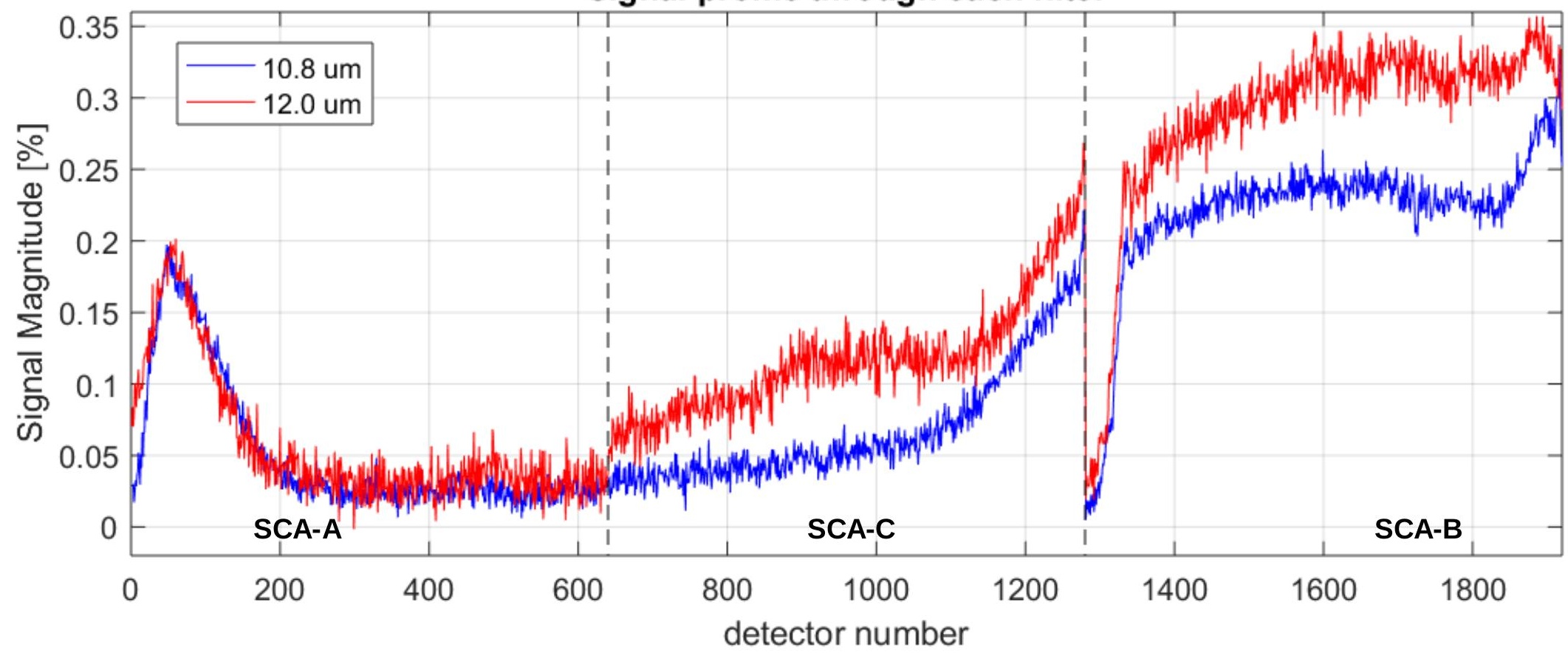




\section{Scatter Results: TIPCE3 Scatter vs. Optical Model}

Optical model from June Tveekrem for SCA-B, 12 um band

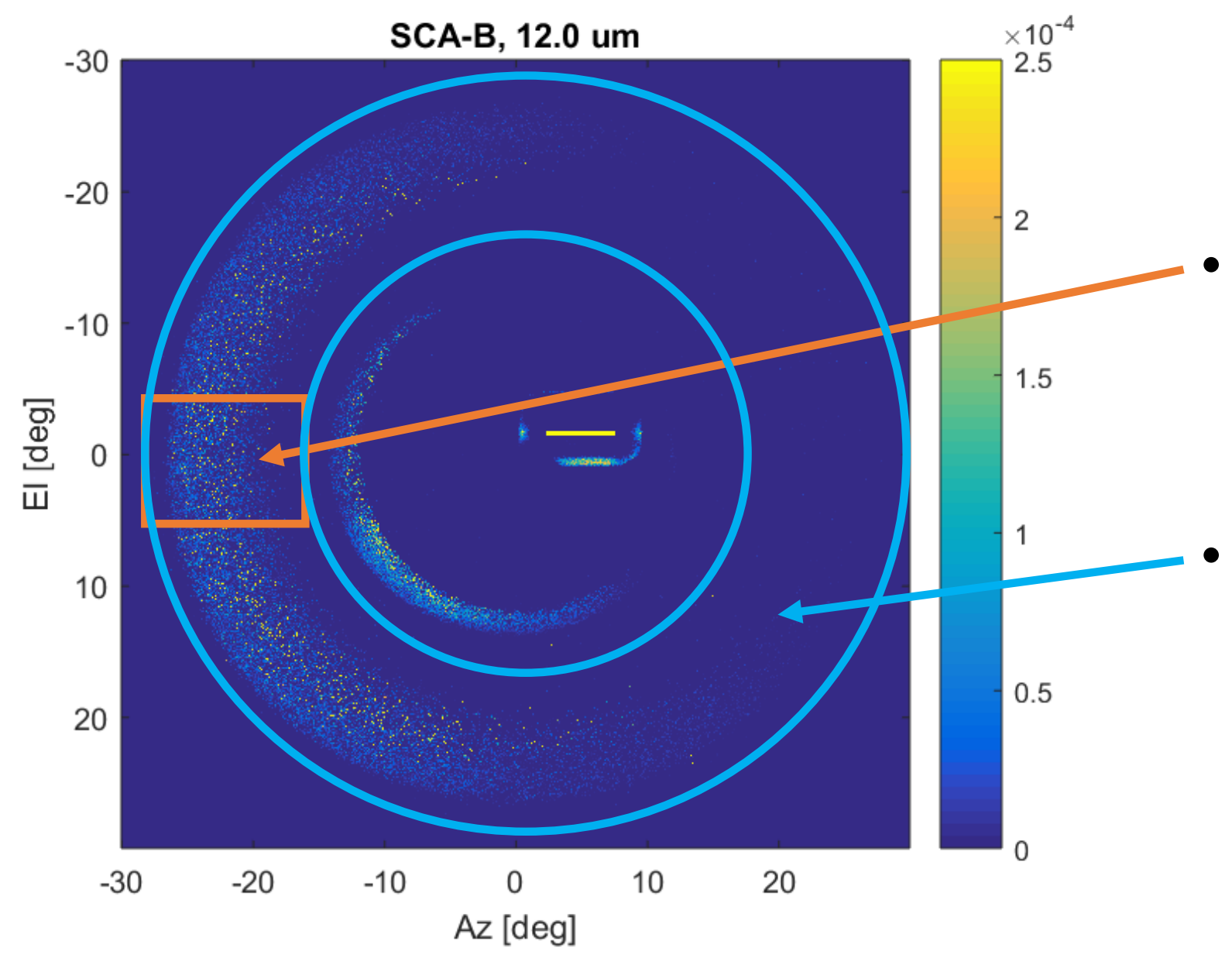

- TIPCE angles do not encompass entire out-offield but can use TIPCE results to scale optical model to same units.

Use sum of TIPCE signal here and sum of model signal here to derive scale factor

Scale entire optical model using scale factor and sum up signal for each SCA/band. 


\section{Scatter Results: Total Scatter Sum}

Sum using optical model :

\begin{tabular}{|l|l|l|}
\hline & $\mathbf{1 0 . 8}$ um & $\mathbf{1 2 . 0} \mathbf{u m}$ \\
\hline SCA-A & $0.69 \%$ & $1.11 \%$ \\
\hline SCA-B & $0.76 \%$ & $1.01 \%$ \\
\hline SCA-C & $0.24 \%$ & $0.21 \%$ \\
\hline
\end{tabular}

Preliminary look at science impact:

SCA-A, 12.0 um, 1.11\%

TIRS-2 estimated to have more than an order of magnitude lower stray light impact than TIRS-1

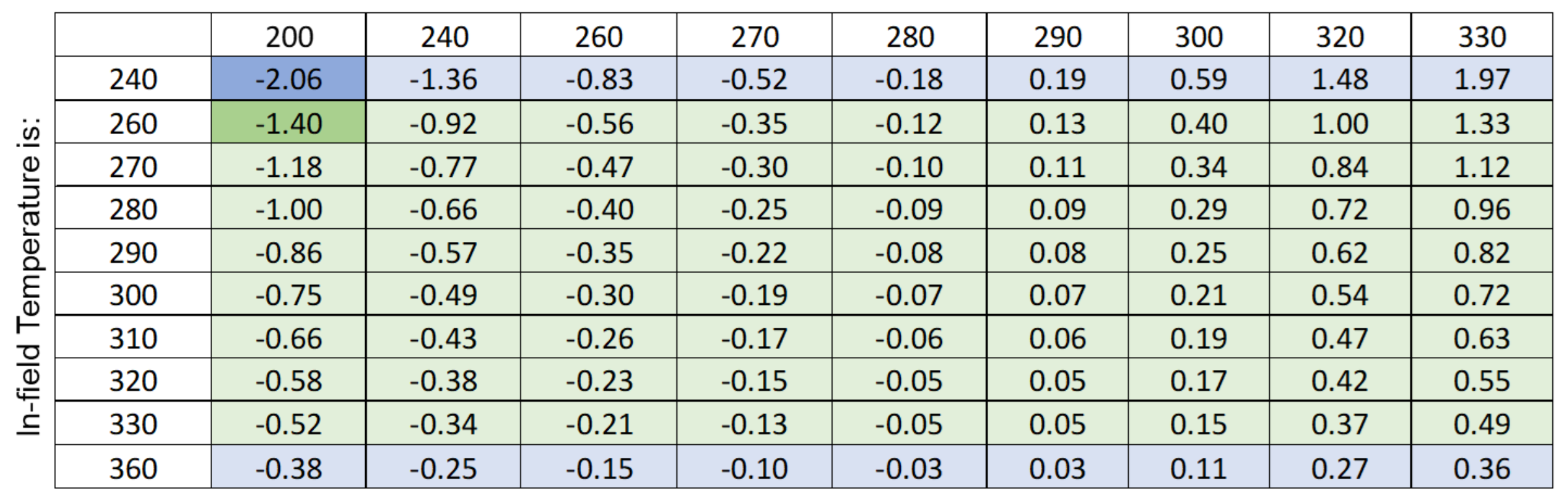

Numbers in table are the percent radiance that the condition is high or low when an out-of-field radiance of $285 \mathrm{~K}$ is assumed and removed from the calibration. 


\section{Spectral Response Test Methodology}

- Data collect with TIRS from the monochromator bracketed by collects with the MCT reference detector

- Cal GSE in "monochromator mode" where collimated beam from the setup outside the chamber is focused and then re-collimated
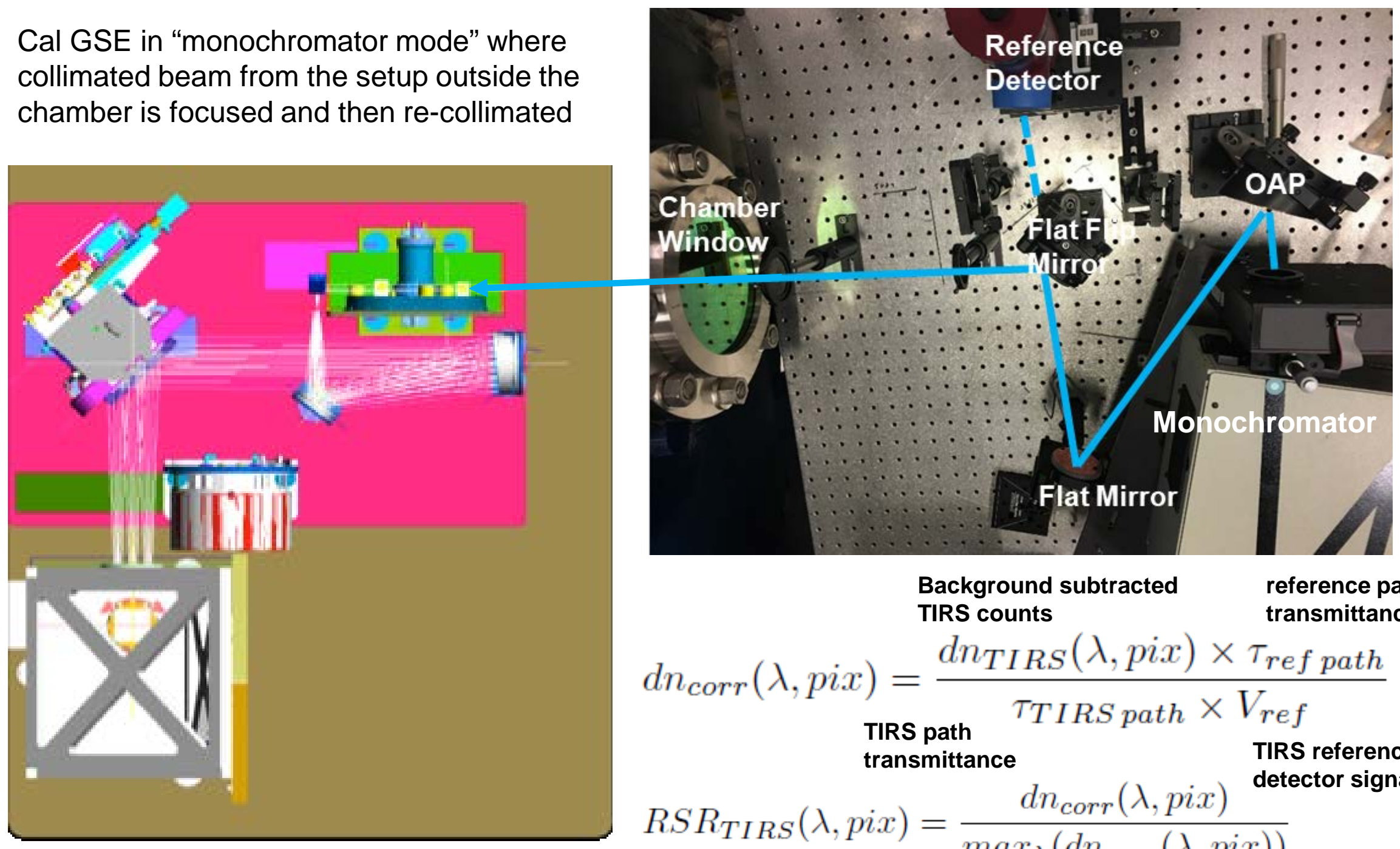

$$
\begin{aligned}
& \text { Background subtracted } \\
& \text { TIRS counts } \\
& d n_{\text {corr }}(\lambda, \text { pix })=\frac{d n_{\text {TIRS }}(\lambda, \text { pix }) \times \tau_{\text {ref path }}}{\tau_{\text {TIRS path }} \times V_{\text {ref }}} \\
& \operatorname{RSR}_{\text {TIRS }}(\lambda, \text { pix })=\frac{d n_{\text {corr }}(\lambda, \text { pix })^{\text {detectemittance }}}{\max _{\lambda}\left(d n_{\text {corr }}(\lambda, p i x)\right)}
\end{aligned}
$$




\section{Spectral Response Test Methodology}

- Data was collected for three or four locations on each SCA.

- The monochromator slits were $2 \mathrm{~mm}(\sim 150 \mathrm{~nm})$.

- TIRS data is collected using the monochromator shutter to provide background measurement. MCT data is collected between channels/SCAs.

- Optimization of the linear stage is run before each collect.

- Optimized for integration time

\section{$10.8 \mu \mathrm{m}$}
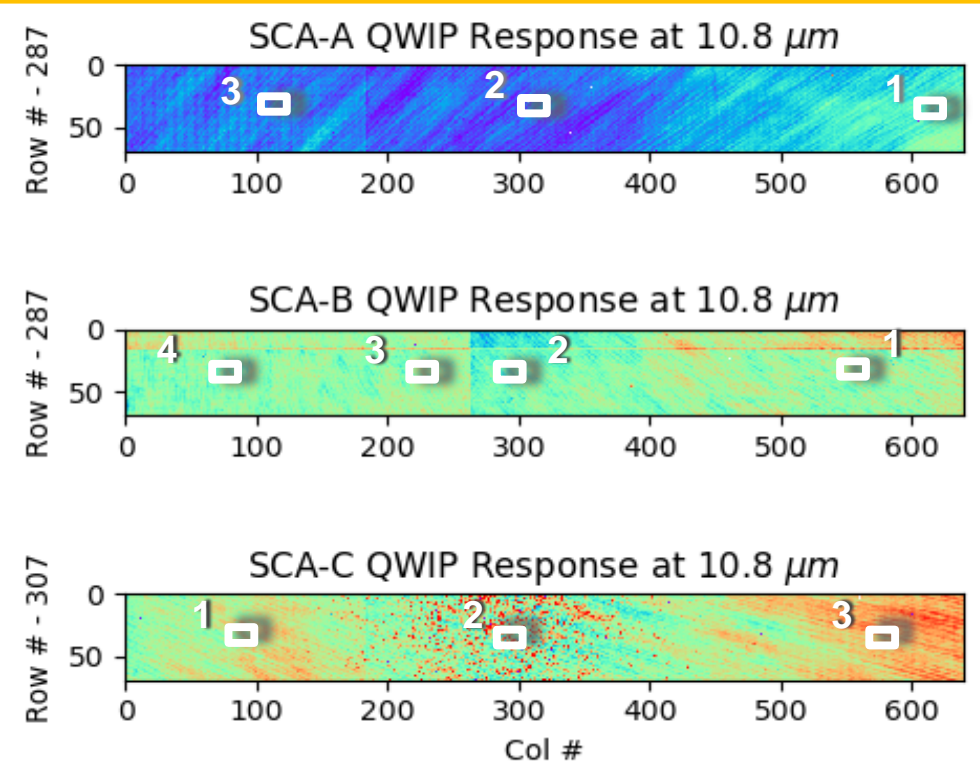

$12.0 \mu \mathrm{m}$

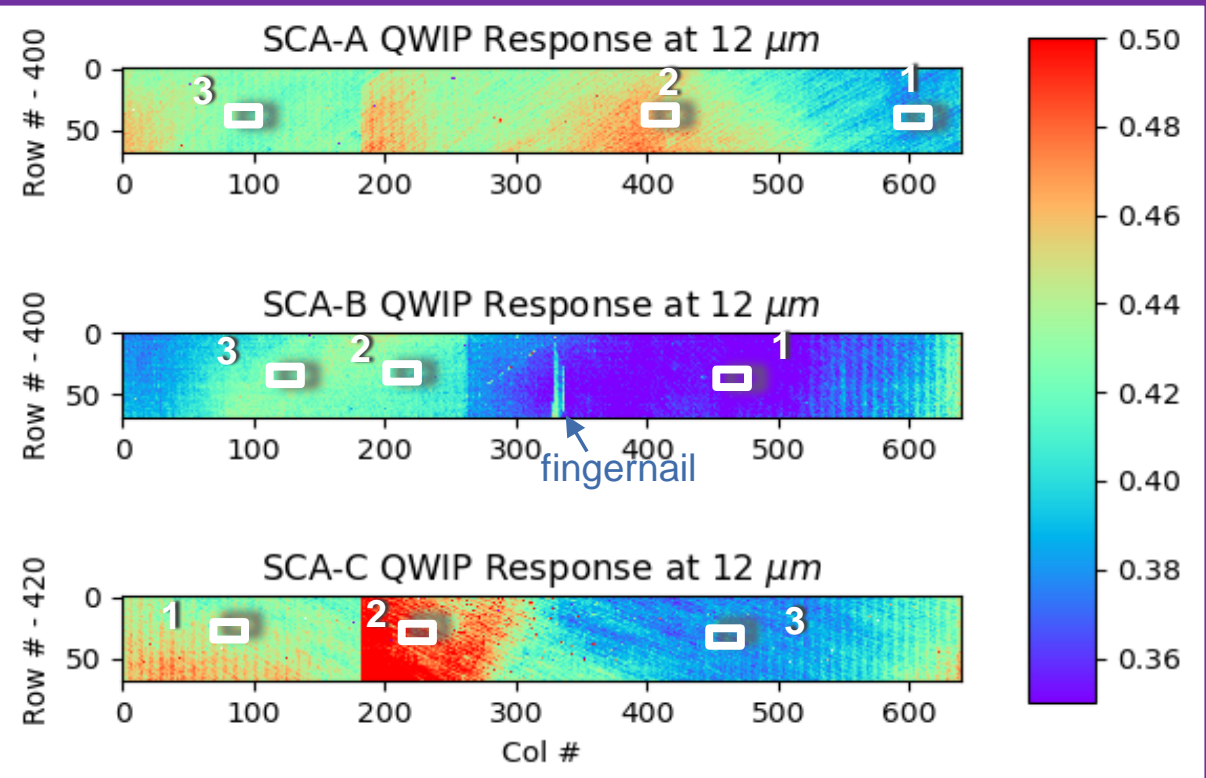




\section{Spectral Response Results}
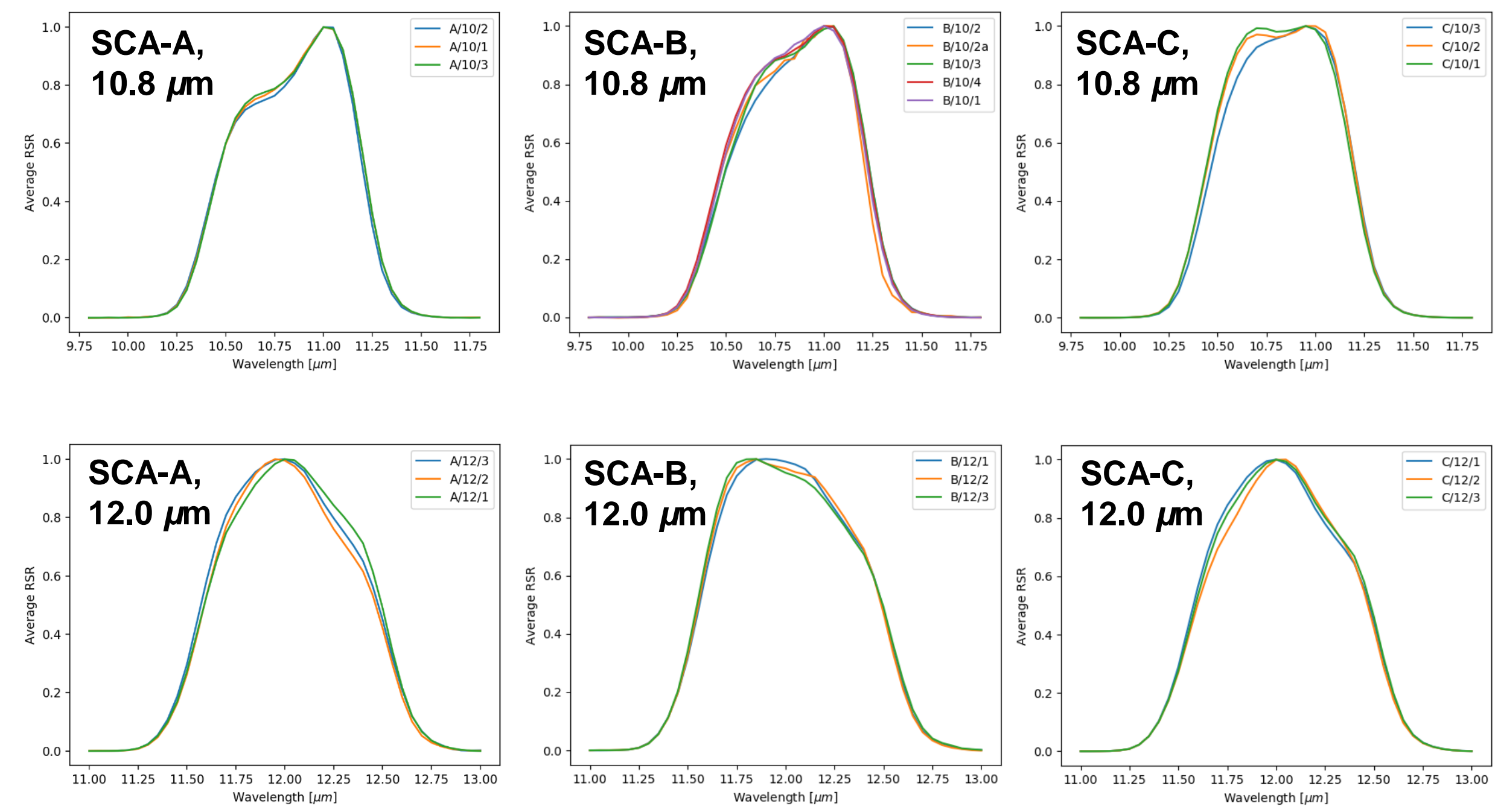


\section{Spectral Response Results: Comparison to Component-Level}
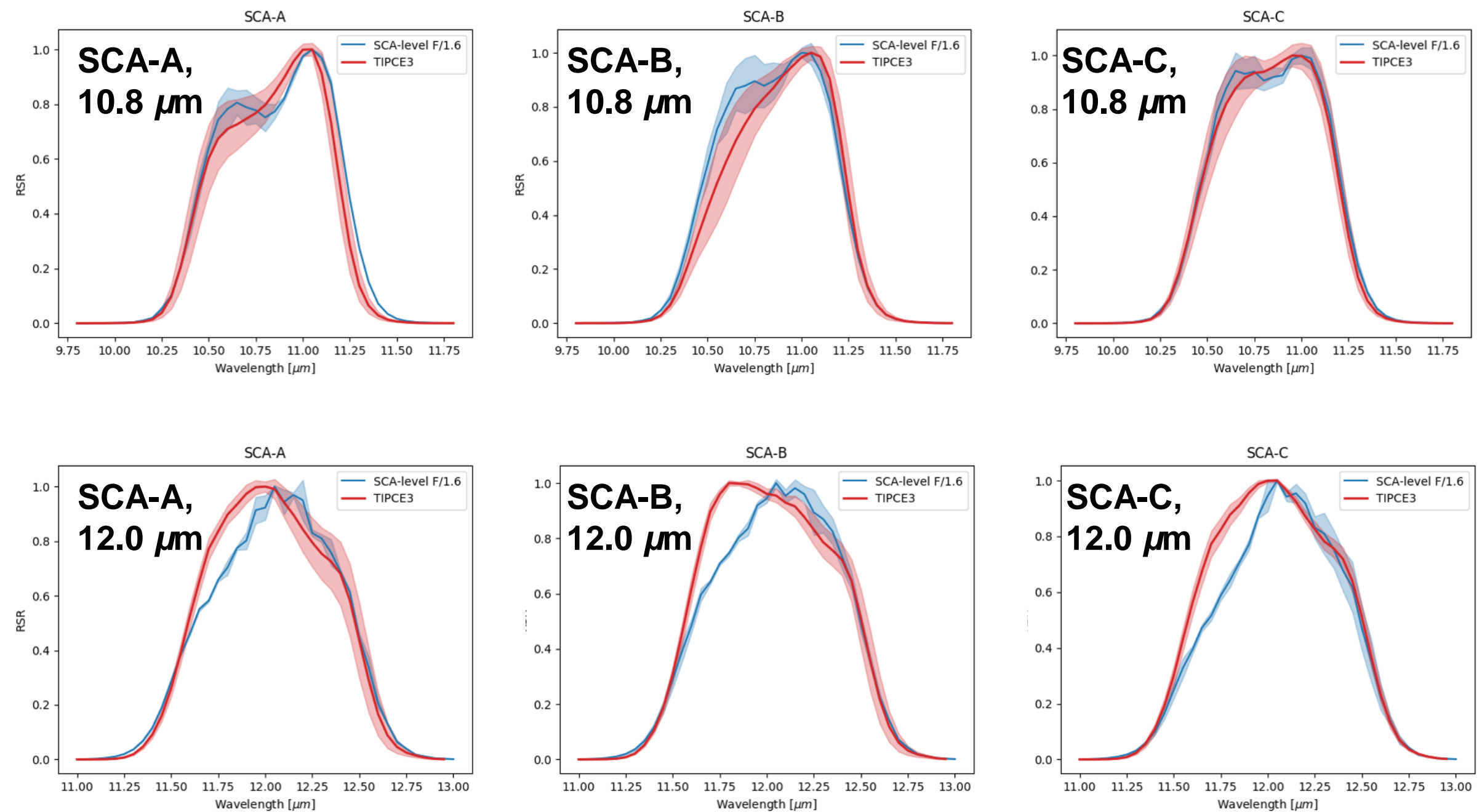

SCA-level (F/1.6) TIPCE

The shading represents the standard deviation over the per pixel RSRs averaged at each location. 


\section{Spectral Response Results: Center Wavelength \& Band Edges}
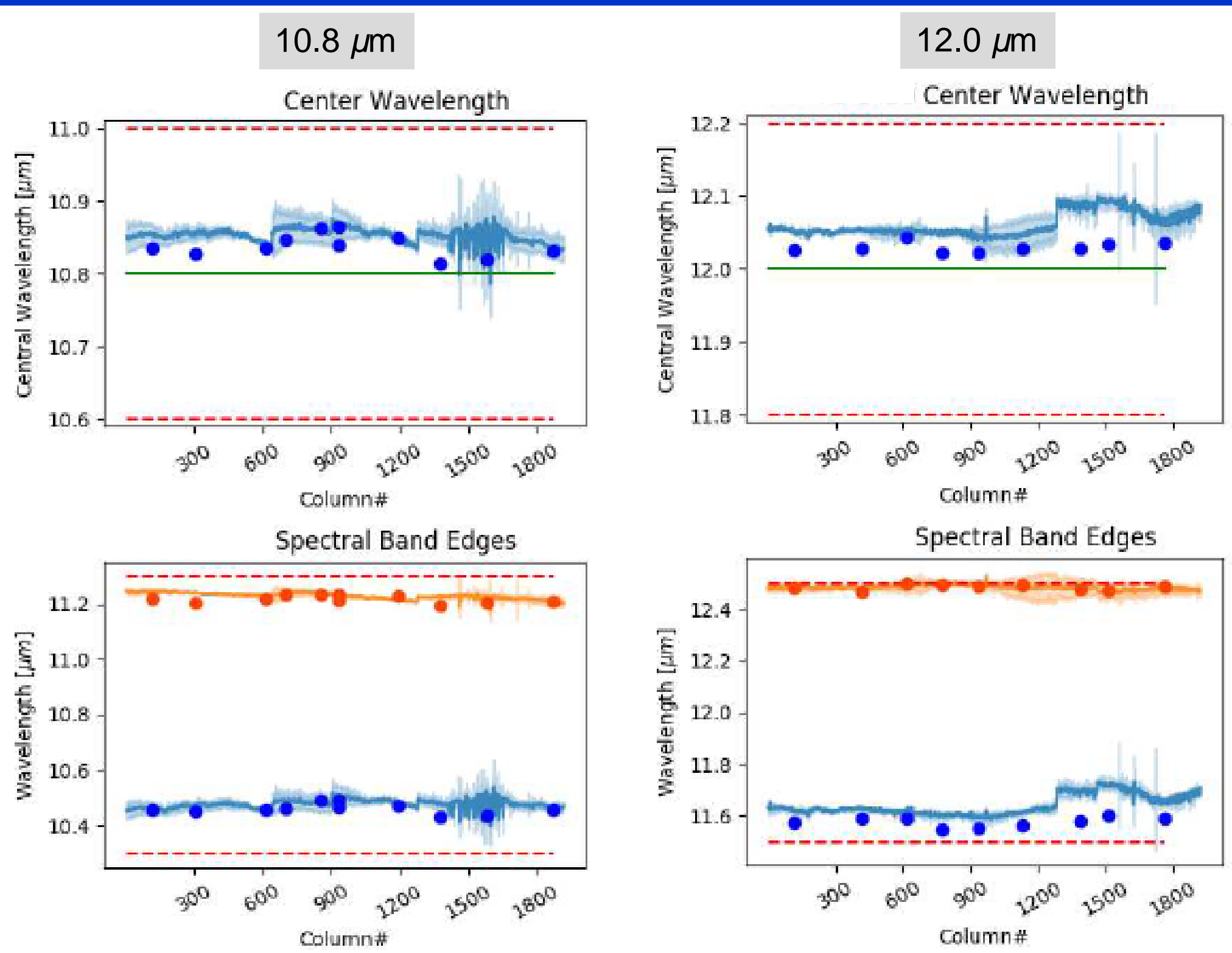


\section{Spectral Response Results: Uniformity}
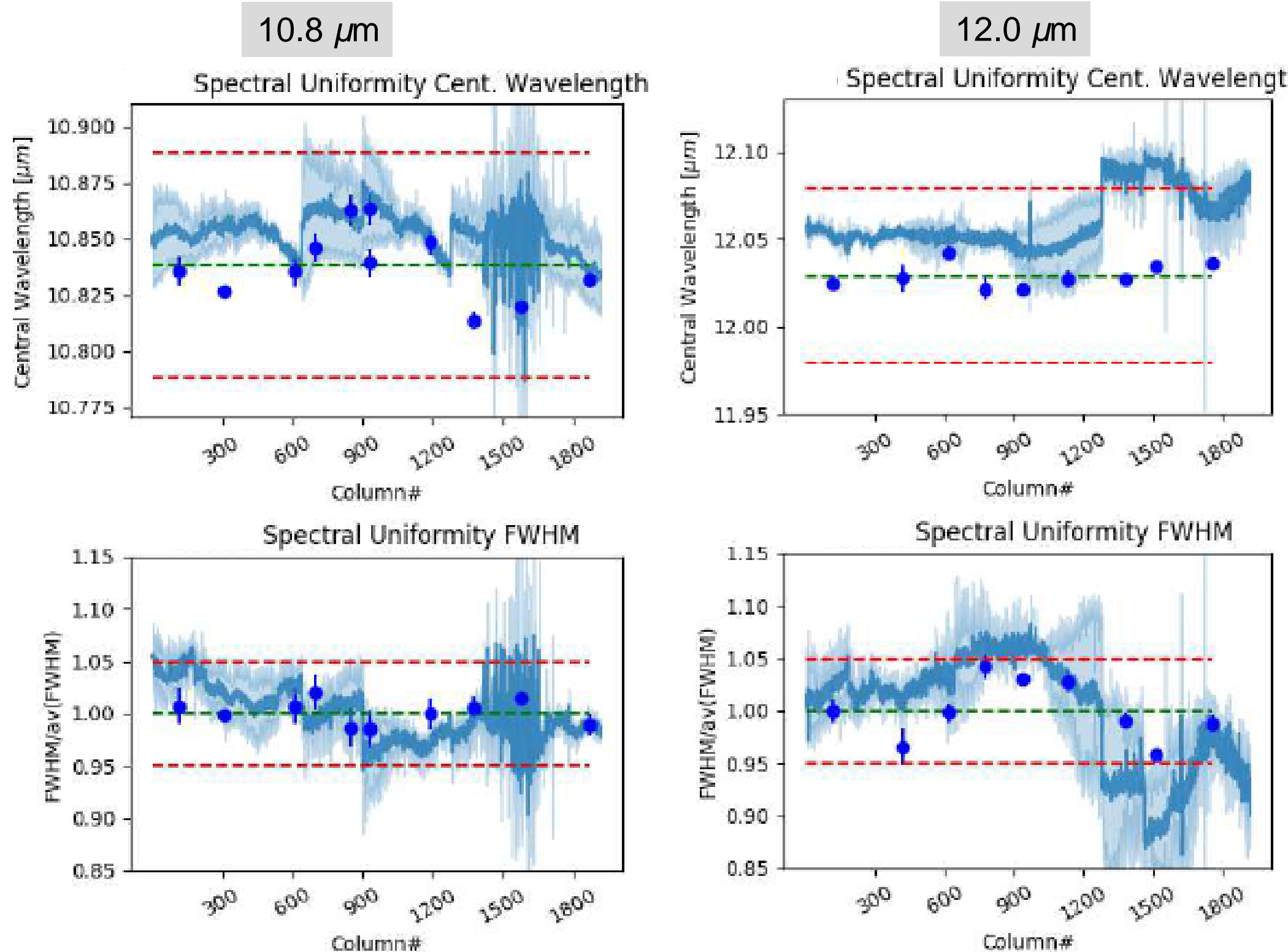


\section{Summary}

- The results show that TIRS-2 performance is expected to meet all of its performance requirements with few waivers and deviations.

- Initial TIRS-2 performance testing set and verified the focus of the instrument.

- Spectral response results show good agreement with component-level measurements accounting for the angular dependence of the detector spectral response.

- The scatter survey showed improved stray light rejection compared to TIRS-1 the total stray light effect of $1 \%$ or less (TIRS-1 - 8\%).

- Current preparations for instrument-level thermal vacuum in the fall testing are now underway and delivery is expected Aug 2019. 


\section{Backup}


- Used NIST wavelength standard (1921b) to calibrate the monochromator wavelength scale using absorption lines closest to the TIRS-2 bands

- The adjustment was programmed into the monochromator to correct an $120 \mathrm{~nm}$ offset before TIPCE

- The wavelength calibration was validated pre/post TIPCE phases

- Monochromator wavelength $<10 \mathrm{~nm}$ from wavelength reference throughout TIPCE.
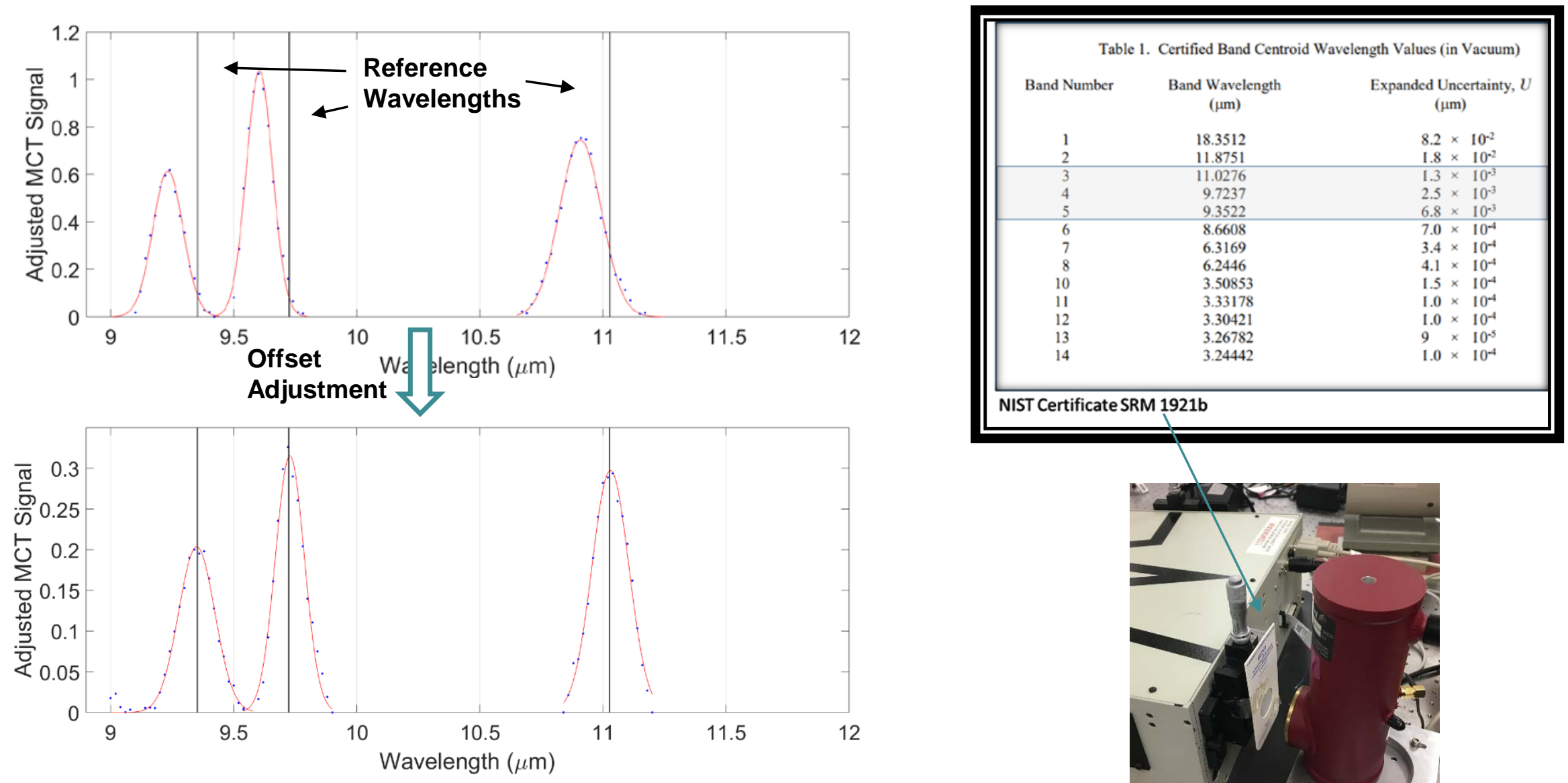


\section{Spectral Shape - Optical Modeling}

Simulated Image on TIRS focal plane

New lens from CVMACRO: cvnewlen irradiance
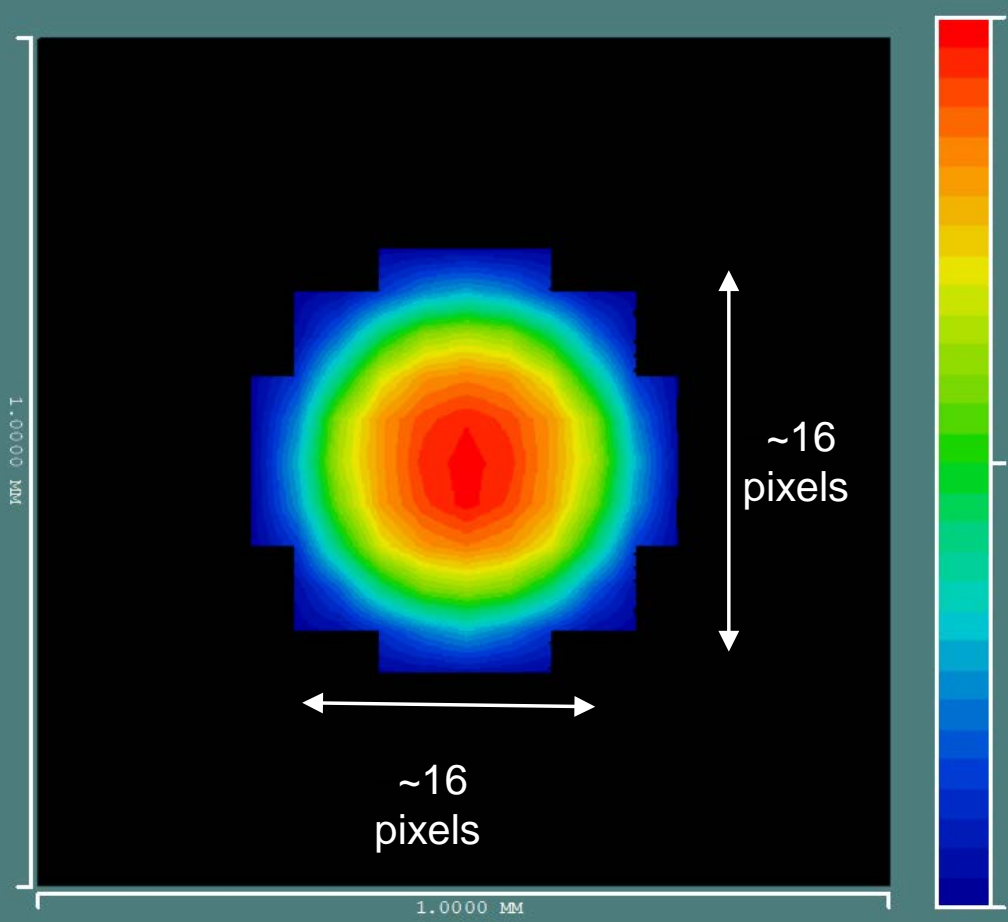

Measured Image on TIRS focal plane

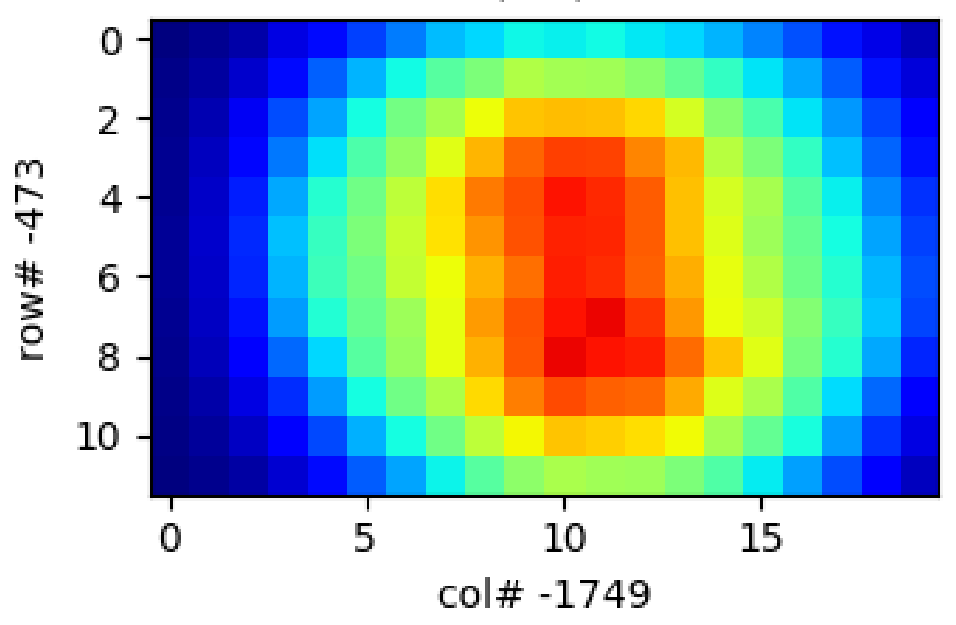

Model and TIPCE show slit images with similar shapes \& sizes 


\section{Thermal Radiance Detected by TIRS-2 from Surface and Atmosphere}

$L_{s}=\frac{\int\left(B(T, \lambda) \cdot \tau(\lambda)+L_{a t m}(\lambda)\right) \cdot R^{\prime}(\lambda) \cdot d \lambda}{\int R^{\prime}(\lambda) \cdot d \lambda}$ $B(T, \lambda) \cdot$ Emitted and reflected surface radiance $\tau(\lambda) \cdot$ Transmission of atmosphere $L_{a t m}(\lambda) \cdot$ Emitted and scattered radiance of atmosphere $R^{\prime}(\lambda) \cdot$ Spectral response of pixel

$L_{s} \cdot$ Pixel integrated radiance

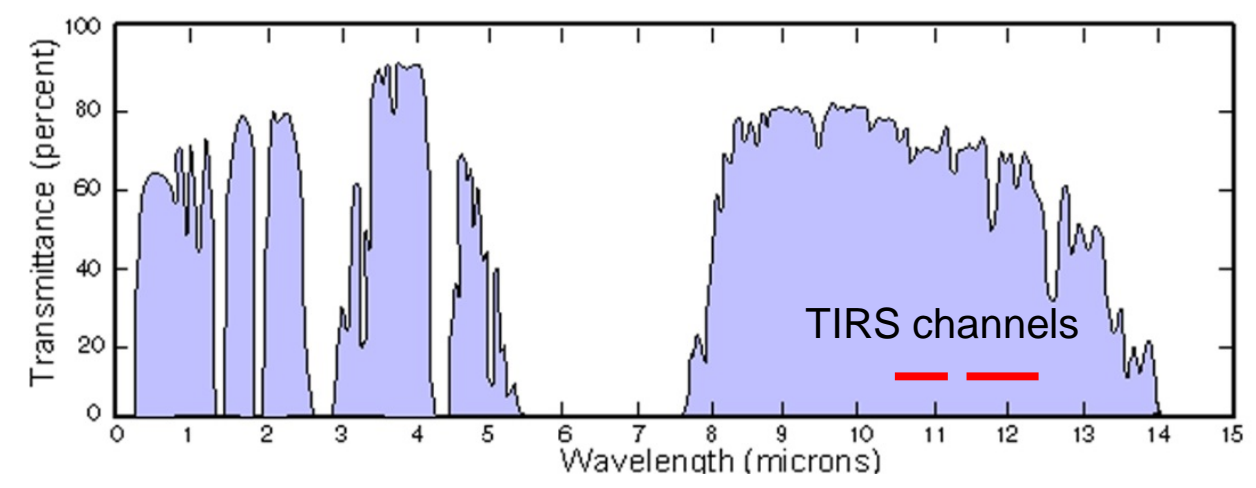

$$
B(T, \lambda)
$$

Two channel "split window" techniques correct for atmosphere and improve retrieved surface temperature 


\section{TIPCE Chamber Configuration}

- Test article consists of major TIRS-2 components (except scene select mirror)

- Front end baffle simulator (FEBSim) forward of telescope to simulate entrance apertures of the optical system

- Test article positioned close to the calibration ground support equipment (Cal GSE) to allow for angular range needed for scatter survey.

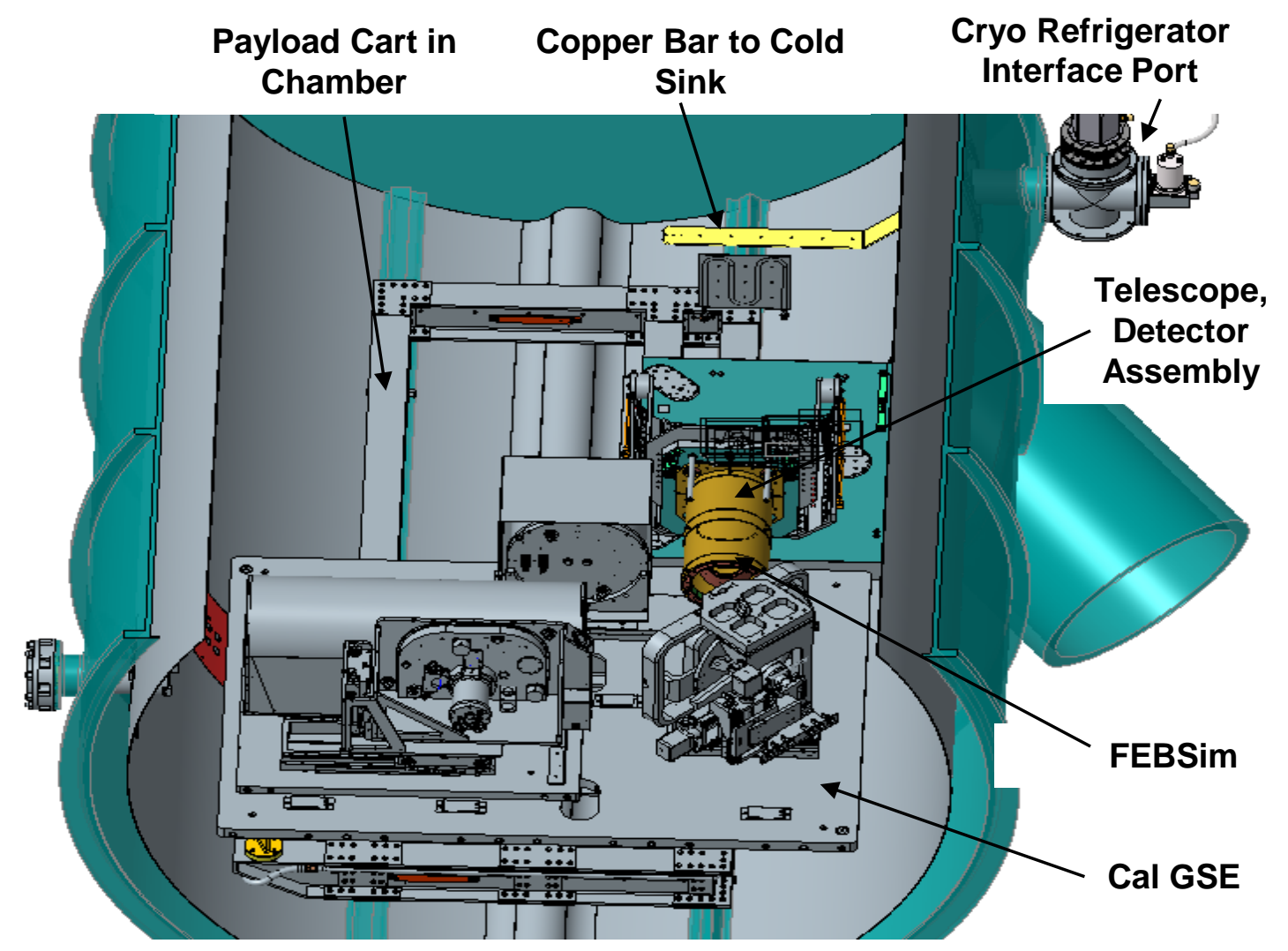

Top Down, Cutaway View of

Thermal Chamber 


\begin{tabular}{|cll|cc|cc} 
& & & \multicolumn{2}{|c|}{ TIRS-2 (TIPCE-3) } & \multicolumn{2}{|c|}{ TIRS Pre-launch } \\
& \multicolumn{1}{c}{ Channel } & Direction & Mean & $\sigma$ & Mean & $\sigma$ \\
\hline \multirow{2}{*}{ Edge Slope $\left(\right.$ pixel $^{-1}$ ) } & $10.8 \mu \mathrm{m}$ & Cross & 0.0059 & 0.0001 & 0.0059 & 0.0002 \\
& $10.8 \mu \mathrm{m}$ & Along & 0.0058 & 0.0002 & 0.0053 & 0.0003 \\
& $12.0 \mu \mathrm{m}$ & Cross & 0.0059 & 0.0001 & 0.0061 & 0.0001 \\
& $12.0 \mu \mathrm{m}$ & Along & 0.0060 & 0.0001 & 0.0063 & 0.0002 \\
Edge Extent $(\mathrm{m})$ & $10.8 \mu \mathrm{m}$ & Cross & 215.6 & 7.3 & 202.8 & 9.1 \\
& $10.8 \mu \mathrm{m}$ & Along & 222.8 & 6.8 & 234.0 & 17.1 \\
& $12.0 \mu \mathrm{m}$ & Cross & 214.9 & 5.1 & 197.6 & 6.9 \\
& $12.0 \mu \mathrm{m}$ & Along & 207.5 & 5.2 & 184.3 & 11 \\
\hline
\end{tabular}

